\title{
Melatonin Enhances Osteoblastogenesis from Senescent Mesenchymal Stem Cells via MMSET Mediated Chromatin Remodeling
}

Zhiqiang Liu ( $\nabla$ zhiqiangliu@tmu.edu.cn )

Tianjin Medical University https://orcid.org/0000-0002-0677-8097

\section{Ying Xie}

Tianjin Medical University

Na Han

Peking University

\section{Sheng Wang}

Tianjin Medical University

\section{Xuelei Wei}

Tianjin Hospital

\section{Jingya Wang}

Tianjin Medical University

\section{Jing Guo}

Tianjin Medical University

Hongmei Jiang

Tianjin Medical University

Jingjing Wang

Tianjin Medical University

\section{Xin Li}

Tianjin Medical University

\section{Xiyun Bian}

The Fifth Central Hospital of Tianjin

\section{Meilin Hu}

Tianjin Medical University

\section{Zhongjiao Zhu}

Tengzhou Central People's Hospital

\section{Lijuan Wang}

Linyi People's Hospital

\section{Hui Zhang}

Children's Hospital of Chongqing Medical University

Chunhua Liu 
Shandong First Medical University \& Shandong Academy of Medical Sciences

\section{Xiaozhi Liu}

The Fifth Central Hospital

\section{Article}

Keywords: melatonin, mesenchymal stem osteoporosis, senescence, MMSET

Posted Date: January 4th, 2021

DOl: https://doi.org/10.21203/rs.3.rs-130548/v1

License: (c) (i) This work is licensed under a Creative Commons Attribution 4.0 International License. Read Full License 
Ying $\mathrm{Xie}^{1^{*}}, \mathrm{Na} \mathrm{Han}{ }^{2 *}$, Feng $\mathrm{Li}^{3 *}$, Sheng Wang ${ }^{1 *}$, Xuelei Wei ${ }^{4}$, Jingya Wang ${ }^{1}$, Jing Guo ${ }^{1}$, Hongmei Jiang ${ }^{1}$, Jing $\mathrm{Liu}^{1}$, Jingjing $\mathrm{Wang}^{1}$, Xin $\mathrm{Li}^{1}$, Xiyun Bian ${ }^{5}$, Meilin $\mathrm{Hu}^{6}$, Zhongjiao

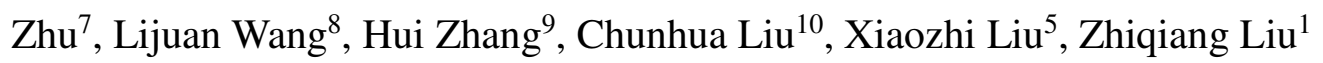

${ }^{1}$ The province and ministry co-sponsored collaborative innovation center for medical epigenetics; Tianjin Key Laboratory of Cellular Homeostasis and Human Diseases; Department of Physiology and Pathophysiology, School of Basic Medical Science, Tianjin Medical University, Heping, Tianjin, China; ${ }^{2}$ Department of Central Laboratory \& Institute of Clinical Molecular Biology, Peking University People's Hospital; National Center for Trauma Medicine, Beijing, China; ${ }^{3}$ Department of Orthopaedics, Weifang People's Hospital, Weifang, Shandong, China; ${ }^{4}$ Department of Emergency, Tianjin Hospital, Hexi, Tianjin, China; ${ }^{5}$ Central Laboratory, The Fifth Central Hospital of Tianjin, Tianjin, China; ${ }^{6}$ Tianjin Medical University School of Stomatology, Heping, Tianjin, China; ${ }^{7}$ Department of Orthopaedics, Tengzhou Central People's Hospital, Tenghzou, Shandong, China; ${ }^{8}$ Department of Hematology, Linyi People's Hospital, Shandong University, Linyi, Shandong Province, China; ${ }^{9}$ Department of Cardiology, Heart Centre; Ministry of Education Key Laboratory of Child Development and Disorders National Clinical Research Center for Child Health and Disorders; Chongqing Key Laboratory of 
Pediatrics; China International Science and Technology Cooperation base of Child Development and Disorders; Children's Hospital of Chongqing Medical University, Chongqing, China; ${ }^{10}$ Department of Physiology, Shandong First Medical University \& Shandong Academy of Medical Sciences, Jinan, Shandong, China

RUNNING TITLE: melatonin stimulates osteoblastogenesis via MMSET

\section{*These authors contribute equally to this work.}

1 Corresponding to: Chunhua Liu, Ph.D., Associate Professor, Department of Physiology,

2 Shandong First Medical University \& Shandong Academy of Medical Sciences, Jinan,

3 Shandong, 271096 China, Email: chhliu@sdfmu.edu.cn; Xiaozhi Liu, M.D., Ph,D.,

4 Professor, Central Laboratory, The Fifth Central Hospital of Tianjin, Tianjin, 300450

5 China, email: 1xz7997@ 126.com; Zhiqiang Liu, M.D., Ph.D., Professor, The province

6 and ministry co-sponsored collaborative innovation center for medical epigenetics;

7 Tianjin Key Laboratory of Cellular Homeostasis and Human Diseases; Department of

8 Physiology and Pathophysiology, School of Basic Medical Sciences, Heping, Tianjin, 9300070 China; Email: zhiqiangliu@ @tmu.edu.cn

10 Abstract: 200

11 Main text: 6,528

12 Figures: 9 
1 Abstract

2 Large numbers of elderly people have aging-associated osteoporosis, but efficient

3 approaches to ameliorate bone loss are limited due to our poor understanding of the

4 underlying mechanisms. In this study, we found that melatonin levels in bone marrow

5 decreased with age, and melatonin primarily enhanced the osteogenic potential of

6 mesenchymal stem cells (MSCs) derived from elderly donors compared with fetal- or

7 young adult-derived MSCs. Mechanistic studies indicated melatonin treatment alleviated

8 the senescence-related hypermethylation of the MMSET promoter, leading to elevated

9 expression of the histone methyltransferase NSD2, and promoted the histone H3

10 dimethylation modification at lysine 36 of the osteogenic genes $R U N X 2$ and

11 SP7/OSTERIX as a consequence. MMSET depletion partially abolished the effects of

12 melatonin on osteogenesis in senescent MSCs in vitro. Moreover, melatonin treatment

13 promoted bone formation and alleviated the progression of osteoporosis in a mouse

14 model of aging. Clinically, severity of senile osteoporosis (SOP) in patients was

15 associated with melatonin levels in bone marrow plasma and the MMSET expression in

16 MSCs, and melatonin treatment enhanced osteoblastogenesis from MSCs derived from

17 SOP patients. Our study discovered a previously unreported epigenetic regulatory role for

18 melatonin in alleviating MSC senescence and suggests that melatonin may be a potent

19 agent for preventing aging-associated osteoporosis.

20 Keywords: melatonin; mesenchymal stem cells; osteoporosis; senescence; MMSET 


\section{Introduction}

2 Melatonin is a neurohormone synthesized and secreted predominantly by the pineal gland

3 under the rhythmic control of the suprachiasmatic nucleus and the light/dark cycle $(1,2)$.

4 Previous studies have shown that melatonin is a key molecule in a wide variety of

5 physiological and pathological processes due to the diverse expression of melatonin and

6 its receptors $(3,4)$. Many of the effects of melatonin are mediated directly through

7 membrane-bound melatonin receptors or indirectly via nuclear orphan receptors of the

8 ROR $\alpha /$ RZR family(5). Accumulating evidence has also indicated that melatonin is

9 involved in bone remolding, osteoporosis, osseointegration of dental implants, and

10 dentine formation(6,7). The aging-related reduction of melatonin levels has been shown

11 to be a crucial factor in bone loss and osteoporosis with aging(8). Osteoporosis is a

12 debilitating chronic disease marked by decreased bone density and strength, resulting in

13 fragile bones(9). The loss of bone among the elderly occurs silently and progressively,

14 without obvious symptoms until a painful fracture occurs. Therefore, serum melatonin

15 levels might be utilized as a biomarker for the early monitoring and prevention of

16 osteoporosis, and a better understanding of the functional machinery of melatonin will

17 benefit the application of melatonin in alleviating the aging-related progression of 18 osteoporosis(8). 
1 Mesenchymal stem cells (MSCs) in bone marrow are multipotent stromal cells with the

2 ability to differentiate into a variety of osteogenic, chondrogenic, adipogenic, or

3 myogenic lineages(10). Melatonin can modulate multiple signals to drive the

4 commitment and differentiation of MSCs into osteoblasts(11,12). Increased oxidative

5 stress and cell injury with aging are causal factors of reduced osteogenesis by MSCs.

6 Numerous studies have confirmed that melatonin can promote osteoblast-like cell

7 proliferation, enhance the expression of type I collagen and bone marker proteins, and

8 facilitate the formation of a mineralized matrix $(13,14)$. A study also suggested that

9 melatonin exerts suppressive effects on osteoclasts via the upregulation of calcitonin

10 secretion by osteocytes(15). Mechanistically, through binding to the MT2 receptor,

11 melatonin elevates the gene expression of bone morphogenetic protein 2 (BMP2), BMP6,

12 alkaline phosphatase $(A L P)$, osteocalcin, and osteoprotegerin to favor osteogenesis, and

13 simultaneously suppresses the receptor activator of NF- $\kappa \mathrm{B}$ ligand pathway to attenuate

14 osteolysis(8). Intriguingly, osteoblasts from $\mathrm{MT}^{-/-}$mice exhibit intrinsic defects in

15 differentiation and mineralization compared with their wild-type counterparts, and the

16 mutant cells fail to respond to melatonin(16). However, despite these known phenotypes

17 and functions of melatonin on osteoblastogenesis, the substantial molecular regulatory

18 mechanisms, especially the epigenetic machinery, are still not well elucidated.

19 Osteoblasts are bone-forming cells derived from MSCs, and the stemness and

20 differentiation properties of MSCs have been shown to decline with age and cellular 
1 senescence(17). Nevertheless, an in-depth understanding of the mechanisms involved in

2 cellular senescence remains elusive, due to the highly intrinsic heterogeneity and

3 complicated genetic or epigenetic regulatory processes in MSCs. Melatonin is an

4 effective agent for the alleviation of apoptotic factors to protect MSCs from cell

5 injury(18). A series of studies conducted by SH Lee and colleagues have demonstrated

6 that melatonin treatment enhances kidney-derived MSC proliferation and prevents cell

7 senescence, probably by upregulating PPAR $\gamma$, via the PrPC-dependent enhancement of

8 mitochondrial function, or by exosomes carrying microRNAs(18-20). A study showed

9 that melatonin can restore the osteoporosis-impaired osteogenic potential of bone

10 marrow-derived MSCs by preserving SIRT1-mediated intracellular antioxidation(21).

11 Moreover, incubation of bone marrow-derived MSCs with melatonin predominantly

12 enhances the expression of BCL2, but decreases the expression of BAX, to protect MSCs

13 from apoptosis(22). Despite these findings, it is unknown whether the beneficial effect of

14 melatonin on maintaining MSC regeneration is based on an aging-associated mechanism.

15 Thus, a thorough understanding of the molecular processes controlling MSC senescence

16 is crucial for identifying the drivers and effectors of age-associated MSC dysfunction and

17 to guide the translational application of MSCs in the clinical setting. In this study, we

18 examined the gene expression profiles of human bone marrow MSCs derived from fetal,

19 young adult, and elderly donors to screen for aging-related genes. We discovered a

20 previously unreported phenotype in MSCs derived from elderly donors, but not from fetal

21 or young adult donors, were more sensitive to melatonin stimulation, at least partially 
1 through the alleviation of DNA methylation on the promoter of the histone

2 methyltransferase MMSET gene. Importantly, melatonin levels in bone marrow plasma

3 were correlated with progression of senile osteoporosis in clinic. Mechanistically,

4 MMSET upregulation facilitated the expression of the osteogenic genes $R U N X 2$ and

5 SP7/OSTERIX by modulating the levels of histone 3 dimethylation at lysine 36 , and the

6 beneficial effect of melatonin against bone loss was confirmed in a mouse model of aging.

7 Thus, our study is the first to report the in-depth epigenetic regulatory mechanism of

8 melatonin on the senescence and osteoblastogenesis-related properties of MSCs.

\section{Methods}

\section{Ethic approval}

11 This study was approved by the Ethic Committee of Tianjin Medical University (No.

12 TMUhMEC2018014), and all the protocols were conformed to the Ethical Guidelines of

13 the World Medical Association Declaration of Helsinki. Signed informed consent was

14 obtained from all participating individuals prior to participation in the study. Animal

15 studies were approved by the Committee on Animal Research and Ethics of Tianjin

16 Medical University (No. TMUaMEC2018001), and the Animal Experiments Ethics

17 Committee of the Fifth Central Hospital of Tianjin (No. TJWZX2018047). All protocols

18 conformed to the Guidelines for Ethical Conduct in the Care and Use of Nonhuman

19 Animals in Research. 


\section{Isolation and culture of bone marrow-derived MSCs}

2 For MSCs isolation from young $(n=12,17-44$ years old, median=25, 10 males and 2

3 females) and elderly healthy donors $(n=12,65-82$ years old, median $=68.5,10$ males and 2

4 females) or osteoporosis patients $(\mathrm{n}=12,74-90$ years old, median $=79$, all male), 3-5 mL

5 of bone marrow biopsies were diluted up to $10 \mathrm{~mL}$ with high-glucose Dulbecco's

6 modified Eagle medium (DMEM) (Gibco, Life Technologies, Carlsbad, CA, USA) and

7 gently loaded onto the top of $10 \mathrm{~mL}$ Ficoll Paque Plus (GE Healthcare, Wauwatosa,

8 Wisconsin, USA) and cells were then fractionated on a lymphoprep density gradient by

9 centrifugation at $800 \times \mathrm{g}$ for 25 minutes at room temperature with the acceleration at 1 .

10 After centrifuge, interfacial mononuclear cells were collected, and washed with

11 phosphatebuffered saline (PBS) at $300 \times \mathrm{g}$ for 10 minutes at room temperature twice,

12 resuspended in DMEM supplemented with $10 \%$ fetal bovine serum (FBS) (Gibco, Life

13 Technologies, Carlsbad, CA, USA), $100 \mathrm{U} / \mathrm{mL}$ of penicillin, $100 \mu \mathrm{g} / \mathrm{mL}$ of streptomycin,

14 and $2 \mathrm{mM}$ L-glutamine (Gibco, Life Technologies, Carlsbad, CA, USA), seeded, and

15 incubated at $37^{\circ} \mathrm{C} / 5 \% \mathrm{CO} 2$. For MSCs isolation from femur of human fetuses $(\mathrm{n}=12$,

$16 \quad 16-22$ weeks, median=18.5, 7 males and 5 females), bone marrow was cultured directly

17 in culture media. After 48 hours, nonadherent cells were removed by changing the

18 medium. Thereafter, the medium was changed every two days. When the cells reached

$1985 \%-95 \%$ confluence, they were trypsinized, counted, and plated again. Cells from

20 passages 3-6 were used for the experiments. 


\section{Isolation of mitochondria from MSC cells}

2 Mitochondrial mRNA was isolated accordingly to previous report(23). Briefly, MSC cells

3 were digested with Trypsin-EDTA Solution and washed twice with precooled PBS. The

4 cells were suspended in the mitochondrial isolation buffer (Mitochondria Isolation Kit for

5 Cultured Cells, Beyotime, C3601) and placed on ice for 15 minutes, homogenized for 15

6 times, then centrifuged at $600 \mathrm{~g}$ for 10 minutes at $4^{\circ} \mathrm{C}$. The supernatant was further

7 centrifuged at $11,000 \times \mathrm{g}$ for $10 \mathrm{~min}$ at $4{ }^{\circ} \mathrm{C}$, and the precipitated mitochondria were used

8 for mitochondrial RNA extraction assay using Trizol (Life Technologies, South San

9 Francisco, CA USA).

\section{Transfection, virus package and infection}

11 Transient transfections to HEK293T cells were performed using polyethyleneimine (PEI)

12 (ThermoFisher Scientific, Carlsbad, CA, USA) in the OPTI-MEM medium (Life

13 Technologies, Carlsbad, CA, USA) with a ratio of 1: 4 to 1: 6 of DNA: PEI. Viral 14 particles were produced by HEK293T cells in a $10 \mathrm{~cm}$ dish transfected with $4 \mu \mathrm{g} \mathrm{PMD}_{2} \mathrm{G}$ 15 and $6 \mu \mathrm{g} \mathrm{psPAX}_{2}$ packaging plasmids (Addgene, Watertown, MA, USA), together with $16 \quad 8 \mu \mathrm{g}$ lentiviral expressing vectors encoding target genes, including

17 pCMV3-C-HA-MMSET, pLKO.1 vector encoding shRNAs targeting MMSET gene or

$18 D N M T 3 b$ gene. Supernatant carrying the viral particles was harvested 60 hours after

19 transfection and concentrated to $100 \times$ volume by Poly (ethylene glycol) 8,000

20 (Sigma-Aldrich, St. Louis, MS, USA). 
1 For viral infection, $2 \times 10^{5}$ MSCs were seeded in $1 \mathrm{~mL}$ new complete media for 6 hours

2 and then added $50 \mu \mathrm{L}$ viral concentration and $8 \mu \mathrm{g} / \mathrm{mL}$ polybrene, and cells were spin at

$31800 \mathrm{rpm}$ for 45 minutes at $20^{\circ} \mathrm{C}$. 12 hours after spinfection, the medium was changed

4 and cells were cultured for another 48 hours until further management.

\section{$5 \quad$ Senescence $\boldsymbol{\beta}$-Galactosidase Staining}

6 MSCs were cultured in 6-well plate for indicated time, then culture medium were

7 removed and washed once with $2 \mathrm{~mL}$ of $1 \times$ PBS. After cells were fixed with $1 \mathrm{~mL}$ of

8 fixative solution for $15 \mathrm{~min}$ at room temperature and washed three times with $2 \mathrm{~mL}$ of $1 \times$

$9 \quad$ PBS, $1 \mathrm{~mL}$ of the staining solution was added to incubate cells at $37^{\circ} \mathrm{C}$ for hours until

10 chromogenic reaction achieved.

\section{Osteogenesis induction in vitro}

12 MSCs were cultured in a 6-well plate with complete medium. After reaching $80 \%$

13 confluence, the medium was changed to osteogenic differentiation medium in presence or

14 absence of $1 \mu \mathrm{mol} / \mathrm{L}$ melatonin for 14 days with a medium change every 3 days. The

15 osteogenic differentiation medium was composed of high-glucose DMEM, penicillin

$16(100 \mathrm{U} / \mathrm{mL})$, streptomycin $(100 \mu \mathrm{g} / \mathrm{mL})$, dexamethasone $(0.1 \mu \mathrm{mol} / \mathrm{L}), 10 \% \mathrm{FBS}$,

17 ascorbic acid $(50 \mu \mathrm{g} / \mathrm{mL})$, and $\beta$-glycerol phosphate $(10 \mathrm{mmol} / \mathrm{L})$. Osteogenic

18 differentiation was detected by Alizarin Red S staining or alkaline phosphatase assay 19 (ALP). 


\section{$1 \quad$ Alizarin Red S staining quantitation assay}

2 The culture medium was aspirated and the cells were washed three times with PBS. Then,

3 the cells were fixed with fresh $70 \%$ ethanol for 60 minutes at $4^{\circ} \mathrm{C}$ or fresh $95 \%$ ethanol

4 for 10 minutes at room temperature, washed three times with PBS, and stained with 40

5 mM Alizarin Red S solution, pH 4.2 (Sigma-Aldrich, St. Louis, MS, USA) for 5-10

6 minutes at room temperature. Removed the Alizarin Red S solution and wash it with PBS

7 to stop the color reaction. The images were captured with a visible light microscope. To

8 quantify the Alizarin Red staining intensity, stained cells were incubated with a 10\% (w/v)

9 solution of cetyl pyridinium chloride (Sigma-Aldrich, St. Louis, MS, USA) in $10 \mathrm{mmol} / \mathrm{L}$

10 Na-phosphate buffer $(\mathrm{pH} 7.0)$ for 15 minutes at room temperature, then $200 \mu \mathrm{L}$ of

11 supernatant was transferred to a 96-well plate and read by measuring the absorbance at

$12562 \mathrm{~nm}$ with a microplate reader. Each experiment was performed in triplicate.

\section{Alkaline phosphatase assay and quantification}

14 Osteogenic differentiation was detected by Alkaline Phosphatase staining. Briefly, the

15 culture medium was aspirated and the cells were fixed with $10 \%$ neutral formalin buffer

16 for 10 minutes, washed three times with $1 \times \mathrm{PBS}$, and stained with alkaline phosphatase

17 dyeing working solution (Beyotime) at room temperature in dark for 10 minutes or longer,

18 until the color developed to the desired depth. Removed the dyeing working solution and

19 wash it with deionized water for 1-2 times to stop the color reaction. The images were

20 captured with a visible light microscope. To quantify the ALP activity in control and 
1 osteoblast-differentiated MSCs, we used the Alkaline Phosphatase Assay Kit

2 (Colorimetric) (BioVision) with modified protocols. Cells were cultured in under normal

3 or osteogenic induction conditions. On day 7, wells were rinsed once with PBS and were

4 fixed using $3.7 \%$ formaldehyde in $90 \%$ ethanol for 30 seconds at room temperature; then

5 fixative was removed and $50 \mu \mathrm{L}$ of p-nitrophenyl phosphate solution was added to each

6 well and incubated for 60 minutes in the dark at room temperature until a clear yellow

7 color developed. Reaction was subsequently stopped by adding $20 \mu \mathrm{L}$ of stop solution.

8 Optical density was then measured at 405nm using a SpectraMax/M5 fluorescence

9 spectrophotometer plate reader. The data were then analyzed by evaluating the increase in

10 ALP expression of the treated samples compared to the untreated. Each experiment was 11 performed in triplicate.

\section{ELISA}

13 Melatonin level in bone marrow plasma was measured using a human MT(Melatonin)

14 ELISA Kit (Elabscience, Cat: E-EL-H2016c, Wuhan, China) accordingly. Briefly, bone

15 marrow plasma from young $(n=15$, aging 17-45 years, median $=30,11$ males and 4

16 females) and elderly donors ( $n=24$, aging 56-84 years, median=65.5, 15 males and 9

17 females) were diluted at 1:5 with sample dilution buffer and added into the plate with

18 primary antibody for incubation at $37^{\circ} \mathrm{C}$ for $45 \mathrm{~min}$. Afterward, secondary antibody were

19 prepared accordingly and added into samples at $37^{\circ} \mathrm{C}$ for $30 \mathrm{~min}$. Then, the substrate was 
1 added to develop the signal for detection. Determine the optical density (OD value) of

2 each well at once with a micro-plate reader set to $450 \mathrm{~nm}$.

\section{Quantitative reverse transcription polymerase chain reaction (qRT-PCR)}

4 Total RNA was extracted from cells in different treatment groups using Trizol (Life

5 Technologies, South San Francisco, CA USA) and then converted to cDNA using the $5 \times$

6 All-In-One reverse transcription MasterMix (abm, Vancouver, Canada) according to the

7 manufacturer's instructions. qRT-PCR was performed on a QuantStudio 3 Real-Time PCR

8 System (Applied Biosystems) using EvaGreen 2× qPCR MasterMix (abm, Vancouver,

9 Canada). Expression levels of the following genes were analyzed: AANAT, HIOMT,

10 RUNX2, COL1A1, OPN, SP7, BGLAP, MT1, MT2, DNMT1, DNMT3a, DNMT3b and

11 MMSET. The expression level of the glyceraldehyde-3-phosphate dehydrogenase

$12(G A P D H)$ gene served as a reference. The $\mathrm{Ct}$ value of the GAPDH was subtracted from

13 the $\mathrm{Ct}$ value of the target gene $(\Delta \mathrm{Ct})$, and the average $\Delta \mathrm{Ct}$ value of the triplicates was

14 recorded. The relative expression levels of each gene were determined using the $2^{-\Delta \Delta \mathrm{Ct}}$

15 method.

\section{Western blotting}

17 Protein lysates were prepared in RIPA-buffer (50 mM Tris-Hcl, pH 7.5, $150 \mathrm{mM} \mathrm{NaCl}$, $1810 \mathrm{mM}$ EDTA, 0.5\% sodium deoxycholate, 1\% NP-40, $1 \mathrm{mM}$ sodium ovanadate, 10

$19 \mu \mathrm{g} / \mathrm{mL}$ aprotinin, $1 \mathrm{mM}$ phenylmethanesulfonyl fluoride, and $10 \mu \mathrm{g} / \mathrm{mL}$ leupeptin)

20 supplemented with complete protease inhibitors (Roche, Indianapolis, IN, USA). The 
1 protein concentration was determined using the BCA protein assay kit (ThermoFisher

2 Scientific, Carlsbad, CA, USA). Cell lysate $(50 \mu \mathrm{g})$ was separated by electrophoresis on

3 SDS-PAGE gel and transferred to nitrocellulose membranes (Pall Corporation,

4 Washington, NY, USA). Membranes were blocked with 5\% non-fat milk for 1 hour at

5 room temperature and probed overnight at $4{ }^{\circ} \mathrm{C}$ with specific antibodies. Antibodies used

6 in this study were listed in the supplementary resources. Membranes were washed three

7 times in PBST the next day, then incubated with horseradish peroxidase-conjugated

8 secondary antibodies for 1 hour at room temperature, washed three times with PBST and

9 finally bands were visualized using an enhanced chemiluminescence system (Millipore,

10 Los Angeles, CA USA). The representative Western blot images for at least three

11 independent experiments shown in the figures have been cropped and auto contrasted.

12 Chromatin-immunoprecipitation (ChIP), ChIP-qPCR and ChIP-sequencing

\section{3 (ChIP-seq)}

1420 million cells were washed in PBS and cross-linked with $1 \%$ formaldehyde for 10

15 minutes at room temperature and then quenched by addition of glycine $(125 \mathrm{mM}$ final

16 concentration) for 5 minutes. For Nuclei isolation, cells were resuspended in cell lysis

17 buffer (50 mM Tris, pH 8.0, $140 \mathrm{mM} \mathrm{NaCl,} 1 \mathrm{mM}$ EDTA, 10\% glycerol, 0.5\% NP-40,

$180.25 \%$ Triton X-100), incubated the tube on ice for 20 minutes to swell. Harvested the

19 nuclei by centrifugation at $2000 \mathrm{~g}$ for 5 minutes at $4^{\circ} \mathrm{C}$ resuspended in $1 \mathrm{~mL}$ ChIP lysis

20 buffer (1\% SDS, $10 \mathrm{mM}$ EDTA, $50 \mathrm{mM}$ Tris-HCl, $\mathrm{pH}$ 8.0) and incubated on ice for 10 
1 minutes. Chromatin was fragmented to 200-500 bp using 10 cycles using the Vibra-Cell

2 Ultrasonic Liquid Processors (SONICS, Newtown, CT, USA). For each IP, chromatin

3 was immunoprecipitated with $2 \mu \mathrm{g}$ of antibody in IP dilution buffer (1\% Triton X-100, 2

$4 \mathrm{mM}$ EDTA, $150 \mathrm{mM} \mathrm{NaCl}, 20 \mathrm{mM}$ Tris- $\mathrm{HCl}, \mathrm{pH} 8.0)$ at $4^{\circ} \mathrm{C}$ overnight. Chromatin was

5 precleared for 2 hours each with protein G agarose beads (Cell Signaling Technology,

6 Danvers, MA, USA) before immunoprecipitation. The immunoprecipitated material was

7 washed, once in TSE I buffer (20 mM Tris-HCl, pH 8.0, 2 mM EDTA, pH 8.0, $150 \mathrm{mM}$

$8 \mathrm{NaCl}, 1 \%$ Triton $\mathrm{X}-100,0.1 \%$ SDS $)$, once in TSE II buffer (20 mM Tris-HCl, pH 8.0, 2

9 mM EDTA, pH 8.0, $500 \mathrm{mM} \mathrm{NaCl}, 1 \%$ Triton X-100, 0.1\% SDS), once in LiCl buffer

10 (10 mM Tris-HCl, pH 8.1, $250 \mathrm{mM} \mathrm{LiCl,} \mathrm{1 \%} \mathrm{deoxycholate,} \mathrm{1 \%} \mathrm{NP40,} \mathrm{1mM} \mathrm{EDTA)} \mathrm{and}$

11 once in TE buffer (10 mM Tris, $\mathrm{pH} 8.0,1 \mathrm{mM}$ EDTA, $\mathrm{pH}$ 8.0) before elution in elution

12 buffer (100 mM NaHCO3, 1\% SDS). The samples were removed from beads, reversed

13 cross-linked overnight at $65^{\circ} \mathrm{C}$ and DNA was isolated using QIAquick PCR Purification

14 Kit (QIAGEN, Germantown, MD, USA). Precipitated DNA was analyzed by qPCR or

15 high-throughput sequencing (Novogene Co., Ltd.). Antibodies used in this study were

16 listed in the supplementary resources.

\section{Gene expression microarray}

18 Total RNA was extracted from MSC cells of different age groups using TRIzol reagent

19 (Life Technologies, South San Francisco, CA USA) according to the manufacturer's

20 instructions. Quality of the purified RNA was tested on Agilent 2100 Bio analyzer 
1 (Agilent RNA 6000 Nano Kit) (BGI, Shenzhen, China). Libraries for cluster generation

2 and DNA sequencing were prepared following an adapted method from BGISEQ-500

3 platform. The low quality reads (more than $20 \%$ of the bases qualities are lower than 10)

4 were filtered to get the clean reads. Then those clean reads were assembled into Unigenes,

5 followed with Unigene functional annotation, SSR detection and calculate the Unigene

6 expression levels and SNPs of each sample. Finally, DEGs (differential expressed genes)

7 were identified between samples and do clustering analysis and functional annotations.

8 Reduced representation bisulfite sequencing (RRBS)

9 DNA was extracted from MSC cells of different age groups using Roche kit according to

10 the manufacturer's instructions. Digesting DNA using the $M s p I$ restriction enzyme, which

11 cuts DNA at its recognition site (C $\downarrow \mathrm{CGG}$ ) independent of the $\mathrm{CpG}$ methylation status,

12 then, repairing the end and ligating adapters for Illumina sequencing, selecting gel-based

13 DNA fragments with insert sizes ranging from $160 \mathrm{bp}$ to $400 \mathrm{bp}$, bisulfite treated two

14 successive rounds, after which we observed $98 \%$ converted cytosines outside the CpGs,

15 the bisulfite-converted library was used to PCR amplification for 20 cycles, finally,

16 single-read sequencing for 76 cycles using an Illumina Genome Analyzer II.

\section{Animal experiment and bone morphology in vivo}

18 Aging C57 mice (18 months old) and adult C57 mice (2 months old) were blindly

19 randomized to mock group and melatonin treatment group, and treated with vehicle or 10

$20 \mathrm{mg} / \mathrm{kg}$ melatonin respectively by subcutaneous injection for 10 weeks, twice a week. 2 
1 weeks before termination, calcein $(0.5 \mathrm{mg} / \mathrm{mice}$, i.p., biw $\times 2)$ was intraperitoneally

2 injected for the last 2 weeks, twice a week. To detect the fluorescence intensity in mouse

3 femur, bone tissues were resin-embedded for hard tissue slides cutting with a $50 \mu \mathrm{m}$

4 thickness, and pictures were taken with a fluorescent microscope.

5 To assess the in vivo bone morphology, high-resolution X-ray microtomography was

6 performed on mice femur with the SkyScan 1276 microtomograph (BrukermicroCT,

7 Kontich, Belgium). After segmentation, the 3D models were constructed with the CtAn

8 software (release 2.5, Skyscan). 3D measurements were obtained with the CtAn software

9 (release 2.5, Skyscan). Trabecular bone analysis was performed on the femur body.

\section{Statistical analysis}

11 Data were shown as mean \pm SD for at least three independent experiments. Differences

12 between groups were determined using paired two-tailed Student's $t$-test or two-way

13 ANOVA. The Least Significant Difference (LSD) was used for posting hoc analysis

14 when treatments are less than 2, and the Tukey's Honest Significant Difference test as the

15 post hoc tests when treatments are $>3$. Pearson correlation test was used to determine the

16 correlations between gene expressions, and survival analysis was done by GraphPad

17 Prism 5.0. A $P$ value less than 0.05 was considered statistically significant. $*, P<0.05$;

$18 * *, P<0.01$, compared with the controls, respectively.

\section{Results}


2 Because melatonin is an important endocrine hormone regulating osteogenesis and bone

3 homeostasis, we measured its levels in bone marrow plasma from donors of different

4 ages using an enzyme-linked immunosorbent assay. The amount of melatonin in bone

5 marrow decreased in an age-associated manner, as the average level of melatonin in

6 donors aged under 45 years was approximately $400 \mathrm{pg} / \mathrm{mL}$, but it was less than 250

$7 \mathrm{pg} / \mathrm{mL}$ in donors over 60 years old (Figure 1a). Meanwhile, we assessed the expression

8 of melatonin receptors in bone marrow-derived MSCs isolated from fetal, young adult,

9 and elderly donors using real-time PCR but did not find significant changes in MT1 or

10 MT2 expression (Figure 1b, 1c). To clarify the impact of synthetic enzymes in melatonin

11 synthesis, we isolated total mRNA or mitochondrial mRNA of MSCs derived from fetal,

12 young adult, and elderly donors, and detected the expressions of two key rate-limiting

13 enzymes, arylalkylamine-N-acetyltransferase and

14 hydroxyindole-O-methyltransferase (HIOMT)(24). Total mRNA levels of AANAT and

15 HIOMT were declined at different ranges, but mitochondrial mRNAs were significantly

16 suppressed in MSCs from the old donors, suggesting a possible reason for melatonin

17 attenuation in bone marrow (Figure 1d, 1e). At the same time, the primary characteristic

18 of senescent cells, the activity of lysosomal $\beta$-galactosidase, increased gradually in MSCs

19 from the fetal, the young and the old donors (Figure 1f, 1g). Seemingly, melatonin levels

20 in bone marrow have a close link with aging-associated osteoporosis. 
$1 \quad$ MMSET expression is suppressed in senescent MSCs

2 To screen for differentially expressed genes related to aging, we compared the differences

3 in the gene expression profiles of bone marrow MSCs derived from fetal, young adult,

4 and elderly donors, using gene chip microarray analysis. Multiple comparisons between

5 the three groups of MSCs showed that there was a larger number of differentially

6 expressed genes between the fetal and elderly groups than between the other groups, with

71835 genes with a greater than 2-fold change in expression; comparisons between the

8 fetal and young adult groups and between elderly and young adult groups identified 994

9 and 325 genes, respectively (Figure 2a). Among the 41 genes that overlapped in all three

10 comparisons, we found that the expression of some genes known to correlate with

11 stemness, such as ATP binding cassette subfamily G member 2 ( $A B C G 2$ ), insulin receptor

12 substrate 1 (IRS1), and suppressor of cytokine signaling 1 (SOCS1), together with

13 MMSET, was greatly downregulated in senescent MSCs (Figure 2b). Real-time PCR

14 confirmed that MMSET was maintained at a relatively high level in MSCs from fetal and

15 young adult donors, while its expression was dramatically reduced in senescent MSCs

16 from elderly donors (Figure 2c). Western blot analysis further revealed that MMSET

17 protein expression was significantly decreased in senescent MSCs (Figure 2d). Taken

18 together, these data indicated that MMSET may be an important factor in

19 aging-associated osteoporosis. 
1 Osteogenic potential of senescent MSCs declines in parallel with MMSET

2 downregulation

3 MSCs isolated from the different age groups were cultured in osteoblast differentiation

4 medium for 14 days. ALP activity was investigated and Alizarin Red S staining was

5 performed to assess capacity for osteoblastogenesis. We found that senescence led to the

6 impaired osteogenic potential of bone marrow-derived MSCs, as indicated by decreased

7 ALP activity and mineralization staining (Figure 3a). Quantification of ALP activity and

8 the number of mineralized nodules also confirmed the gradual decline of osteogenic

9 potential with the senescence of MSCs (Figure 3b, 3c). Meanwhile, the expression of

10 osteogenic markers, including bone gamma-carboxyglutamate protein (BGLAP),

11 osteopontin $(O P N)$, and type I collagen alpha 1 (COL1A1), was reduced in senescent

12 MSCs (Figure 3d). Remarkably, association analysis indicated a strong correlation

13 between ALP levels and MMSET expression (Figure 3e), as well as between RUNX2 and

14 MMSET expression (Figure 3f). Interestingly, MSCs from elderly donors exhibited

15 higher ALP activity and mineralized nodule formation after the induction of osteogenesis

16 if they possessed higher levels of MMSET expression (Figure $\mathbf{3 g}, \mathbf{3 h}, \mathbf{3 i}$ ).

17 Melatonin stimulates MMSET expression in senescent MSCs

18 To explore the relationship between melatonin and MMSET, we treated cultured MSCs

19 from donors of different ages with exogenous melatonin $(1 \mu \mathrm{M})$ and then examined 
1 MMSET expression. We found that MSCs from elderly donors exhibited higher

2 sensitivity to melatonin than those from young adults, but no significant change was

3 observed when compared with fetal MSCs (Figure 4a). Western blot analysis was

4 performed using three primary cells of MSCs derived from donors of each age group, and

5 the results further confirmed the downregulation of MMSET expression in senescent

6 MSCs and revealed that senescent MSCs were more sensitive to melatonin $(1 \mu \mathrm{M})$

7 treatment (Figure 4b). Consistently, exogenous melatonin $(1 \mu \mathrm{M})$ significantly

8 ameliorated the repressive status of H3K36me2 in MSCs from elderly donors, which is

9 the catalytic target of MMSET (Figure 4c). To further ascertain the role of melatonin in

10 the osteogenic differentiation of aged MSCs, we examined the expression of the

11 osteogenic markers RUNX2 and SP7/OSTERIX. Their expression was elevated in

12 senescent MSCs in a dose-dependent manner after treatment with melatonin for $24 \mathrm{~h}$

13 (Figure 4d). These data indicated that MMSET upregulation is a marker of

14 melatonin-mediated osteogenesis in senescent MSCs. Since MMSET is a histone

15 methyltransferase that catalyses H3K36me2, and H3K36 methylation has long been

16 implicated in promoting gene transcription(25), we sought to screen genes regulated by

17 H3K36me2 under the stimulation of melatonin using chromatin immunoprecipitation

18 sequencing (ChIP-seq). No large scale genome-wide changes in H3K36me2 occupancy

19 upon melatonin treatment were observed, however the promoter and other regions

20 including the 3'-and 5'-untranslated region (UTR), non-coding regions were slightly

21 increased by $1 \%$, and intergenic region decreased by $2 \%$ (Figure 4e). Melatonin 
1 treatment slightly increased the genome-wide average H3K36me2 signals (Figure 4f),

2 and track profiles indicated that the change in $\mathrm{H} 3 \mathrm{~K} 36 \mathrm{me} 2$ was occurring in promoter,

3 enhancer, and gene body regions, as seen by the representative tracks of the RUNX2 and

$4 \quad$ SP7 genes (Figure 4g).

5 MMSET favors osteogenic differentiation

6 Previous studies have identified MMSET as a histone methyltransferase that can

7 methylate histones $\mathrm{H} 3$ and $\mathrm{H} 4$. We found that the repressive status of $\mathrm{H} 3 \mathrm{~K} 36 \mathrm{me} 2$ was

8 ameliorated and the activated status of $\mathrm{H} 3 \mathrm{~K} 27 \mathrm{me} 3$ was reduced in MSCs from elderly

9 donors if they expressed MMSET at a high level (Figure 5a), suggesting that elevated

10 MMSET expression is a marker of melatonin-mediated osteogenesis in senescent MSCs.

11 To further ascertain the role of MMSET in the osteogenic differentiation of aged MSCs,

12 we manipulated MMSET expression using a lentivirus carrying short hairpin RNA

13 (shRNA) targeting MMSET or with MMSET overexpression vectors. MMSET knockdown

14 led to a decrease in H3K36me2 levels, whereas MMSET overexpression led to an

15 increase in H3K36me2 levels (Figure 5b). MSCs with MMSET overexpression

16 displayed a stronger potential for osteogenesis. After osteogenic induction, these cells

17 exhibited higher levels of ALP activity, increased numbers of mineralized nodules, and

18 enhanced expression of osteogenic markers compared with their vector control

19 counterparts (Figure 5c, 5d). In contrast, MSCs with MMSET knockdown exhibited

20 lower levels of ALP activity and decreased numbers of mineralized nodules (Figure 5e). 
1 Silencing of MMSET impaired the expression of RUNX2 and SP7/OSTERIX induced by

2 melatonin treatment (Figure 5f). ChIP-qPCR analysis showed that melatonin treatment

3 enhanced the recruitment of H3K36me2 to RUNX2 and SP7/OSTERIX promoters in a

4 dose-dependent manner (Figure 5g), and the recruitment of H3K36me2 to RUNX2 and

5 SP7/OSTERIX promoters was correspondingly altered when MMSET was knocked down

6 or overexpressed compared with the control groups (Figure 5h). Importantly, a high

7 concentration of melatonin failed to enhance the recruitment of H3K36me2 to RUNX2

8 and SP7/OSTERIX promoters after MMSET silencing (Figure 5i). Collectively, these data

9 showed that melatonin favored osteogenic differentiation via the MMSET-mediated

10 modification of $\mathrm{H} 3 \mathrm{~K} 36 \mathrm{me} 2$ on the promoters of osteogenic driver genes.

11 Melatonin alleviates DNA methylation of the MMSET promoter

12 Subsequently, we wished to identify the mechanism underlying the downregulation of

13 MMSET in senescent MSCs. We analyzed the genome-wide methylation profiles of

14 cultured MSCs from donors of different ages using reduced representation bisulfite

15 sequencing (RRBS). Based on our data regarding the differentially methylated regions

16 (DMRs) of osteogenesis gene promoters in each genomic feature group, we performed a

17 relative enrichment analysis to identify the changes in the methylation of genes in MSCs

18 from the different age groups to elaborate the relationship between senescence and the

19 methylation status of genes. Genomic distribution of DMRs according to the genomic

20 features in the RRBS data on the different MSC groups showed that the methylation 
1 levels on $\mathrm{CpG}$ islands (CGis) increased with aging, but there were no significant changes

2 in the gene body, 3'-untranslated region (UTR), 5'-UTR, exons, and intergenic and

3 intronic regions (S Figure 1a). By using the UCSC Genome Browser with the human

$4 \quad$ MMSET gene assembly, we found a 1805-bp DNA sequence with a high GC percentage

$5 \quad(76.5 \%)$ containing 261 CGis in the MMSET promoter, and the DNA methylation status

6 within the CGis increased gradually in MSCs from young adult and elderly donors

7 compared with those from fetal donors (Figure 6a).

8 A previous study indicated that the expression of DNMT1 and DNMT3a decreases in 9 aged cells, whereas the expression of DNMT3b mRNA and protein increases steadily(26).

10 In the present study, we also observed that melatonin $(1 \mu \mathrm{M})$ treatment effectively

11 decreased DNMT3b expression but had no significant effect on DNMT1 and DNMT3a

12 levels (Figure 6b). Indeed, in MSCs from elderly donors, there was a negative

13 correlation between MMSET and DNMT3b expression (Figure 6c). Consequently,

14 treatment of senescent MSCs with the methyltransferase inhibitor 5-aza to induce DNA

15 demethylation increased MMSET levels (Figure 6d). To further ascertain the relationship

16 between DNMT3b and MMSET in aged MSCs, we knocked down DNMT3b expression

17 using shRNA (S Figure 2a). DNMT3b silencing directly rescued the expression of

18 MMSET in aged MSCs, but it did not have an obvious effect on MMSET expression in

19 MSCs treated with an increasing concentration of melatonin (Figure 6e). Notably, only

20 when DNMT3b expression was rescued in the DNMT3b-knockdown MSCs, but not 
1 vector control, could restore the upregulation of MMSET and the osteogenic RUNX2 and

2 SP7 expressions upon melatonin treatment (Figure 6f), consequentially enhancing the

3 MSC derived osteogenesis reflected by significantly augmented Alizarin Red staining

4 and ALP activity (Figure $6 \mathrm{~g}$ and $6 \mathbf{h}, \mathbf{S}$ Figure $2 \mathbf{b}$ and $2 \mathbf{c}$ ). Taken together, our results

5 suggest that the decrease of MMSET levels in senescent MSCs may due to

6 DNMT3b-mediated promoter methylation. Melatonin treatment can modify the

7 epigenetic status of the MMSET promoter via DNMT3b-mediated promoter methylation.

8 Melatonin facilitates the osteogenesis of MSCs derived from aged mice via MMSET

9 We verified the relationship between melatonin and MMSET in aged mice. We first

10 evaluated the effects of melatonin on osteoblast-mediated bone formation in vivo. Aged

11 mice (18 months old) and young adult mice (2 months old) were treated with vehicle or

12 melatonin by subcutaneous injection for 8 weeks, and then injected intraperitoneally with

13 calcein for 2 weeks (Figure 7a). We also assessed the osteogenic potential of MSCs from

14 melatonin-treated aged and young adult mice. MSCs from aged mice clearly exhibited

15 decreased osteogenic differentiation, as evidenced by lower levels of ALP activity and

16 reduced numbers of mineralized nodules (Figure 7b); nevertheless, ALP activity and

17 matrix mineralization intensity were effectively increased in the melatonin-treated group

18 compared with the DMSO-treated control group in aged mice (Figure 7c, 7d).

19 Meanwhile, the decreased expression of MMSET in MSCs from aged mice was

20 significantly increased by melatonin treatment, but those from adult mice demonstrated a 
1 limited enhancement (Figure 7e). Subsequently, the in vivo bone formation rate per bone

2 surface $(\mathrm{BFR} / \mathrm{BS})$ by calcein staining and undecalcified bone sections were imaged and

3 analyzed. The results showed that the bone formation rate was increased in the aged

4 mouse group compared with the young adult mouse group under the same treatment

5 conditions (Figure 7f, 7g). A micro-CT assay showed that melatonin significantly

6 increased the bone mass of aged mice and improved the femoral trabecular

7 microstructure (Figure $\mathbf{7 h}, \mathbf{7 i}$ ). Overall, these data from aged mice also supported the

8 hypothesis that melatonin can ameliorate osteogenesis and bone mass of aged mice, and

9 this effect may depend on the induction of MMSET expression.

10 Melatonin treatment recovers the osteogenic potential of MSCs derived from patients

11 with senile osteoporosis

12 Finally, we investigated the association between MMSET and senile osteoporosis (SOP)

13 in the clinical setting. MSCs were collected from bone biopsy samples from healthy

14 elderly individuals and patients with SOP and every donor was examined by radiography

15 (Figure 8a). We found that MMSET expression was significantly lower in bone marrow

16 MSCs from donors with SOP than in those from age-matched control donors (Figure $\mathbf{8 b}$ ).

17 There was a positive correlation between bone mineral density, as measured with dual

18 energy X-ray absorptiometry, and melatonin levels in bone marrow in patients with SOP

19 (Figure 8c), and between bone mineral density and MMSET expression (Figure 8d).

20 Furthermore, MMSET expression showed a very strong correlation with melatonin levels 
1 in bone marrow (Figure 8e). Accordingly, melatonin $(1 \mu \mathrm{M})$ treatment enhanced MMSET

2 expression in MSCs derived from patients with SOP (Figure 8f), and improved their

3 differentiation efficiency after osteogenic induction, as evidenced by higher levels of ALP

4 activity, increased numbers of mineralized nodules (Figure $\mathbf{8 g}, \mathbf{8 h}$ ), and upregulated

$5 \quad R U N X 2$ and SP7IOSTERIX expression (Figure 8i). These data strongly suggest that

6 MMSET downregulation is a characteristic of aging-associated osteoporosis and imply

7 that melatonin may be an efficient therapeutic agent for patients with SOP.

\section{Discussion}

9 The composition, structure, and function of bone deteriorates as a consequence of aging,

10 thereby increasing the risk of osteoporosis in elderly individuals. In this study, we report

11 that melatonin levels in bone marrow decrease in an aging-related manner, and treatment

12 with melatonin can reverse the impaired osteogenic potential of senescent MSCs through

13 an epigenetic regulatory mechanism on the histone methyltransferase MMSET (Figure 9).

14 Our study therefore supports the use of melatonin as a potent therapeutic agent for the

15 prevention of aging-associated osteoporosis.

16 Melatonin is a hormone that is secreted mainly from the pineal gland during darkness and

17 plays a vital role in circadian rhythms(27). Bone marrow in pinealectomized rats can still

18 produce high levels of melatonin, suggesting a function for melatonin in regulating the

19 bone marrow microenvironment(28). Melatonin is known to modulate bone formation 
1 and osteoblast differentiation of bone marrow-derived MSCs(12). Studies have revealed

2 that melatonin can boost osteoblast differentiation by upregulating osterix protein

3 stability and expression(14), reduce autophagy in high glucose-cultured osteoblasts, and

4 alleviate diabetes-induced osteoporosis by suppressing the ERK pathway(29). In addition,

5 melatonin restores the osteoporosis-impaired osteogenic potential of bone

6 marrow-derived MSCs by preserving SIRT1-mediated intracellular antioxidation(30), and

7 SPRY4 may be partially responsible for the melatonin-mediated osteogenesis of bone

8 marrow-derived MSCs(31). However, it is generally unknown how melatonin promotes

9 the osteogenic potential of bone marrow-derived MSCs undergoing aging. In this study,

10 we found that melatonin in bone marrow was declined with aging, probably due to

11 downregulation of the key rate-limiting enzymes AANAT and HIOMT in mitochondrion,

12 which is consistent with a recent study indicating that AANAT could accelerate aging in a

13 knockout mice model(32). Our study also suggests MMSET (also known as WHSC1 or

14 NSD2) is an important factor mediating the effects of melatonin. Melatonin treatment

15 effectively improved MMSET expression and enhanced the H3K36me2 levels of

16 osteogenic genes in senescent MSCs. As a consequence, ALP activity, matrix

17 mineralization, and the expression of osteogenic markers were increased in the melatonin

18 treatment group. By contrast, RNA interference-mediated MMSET knockdown attenuated

19 melatonin-reversed osteogenic differentiation. MMSET is a histone methyltransferase

20 that can methylate histones H3 and H4. MMSET overexpression correlates with an

21 increase in $\mathrm{H} 3 \mathrm{~K} 36 \mathrm{me} 2$ and a decrease in $\mathrm{H} 3 \mathrm{~K} 27 \mathrm{me} 3$ across the genome, leading to a 
1 looser chromatin structure. MMSET is frequently overexpressed in patients with multiple

2 myeloma and its methyltransferase activity is crucial for clonogenicity(33). However, the

3 relationship between MMSET with aging and osteoporosis has not been reported

4 previously, and this is the first description of the importance of this mediator in MSC

5 senescence.

6 Our results established a link between MMSET expression and aging. We identified

7 about 40 genes whose expressions were altered during the senescence of MSC, most of

8 that are stemness-related or senescence-related, such as CCDC28B, SLC19A1, SOX9,

$9 A B C G 2, N A N O G, R U N X 2$, and $C D K N 2 A$ as expected. The results also revealed that

10 MMSET was greatly decreased in senescent MSCs derived from the bone marrow of

11 elderly donors. Western blot analysis further confirmed that MMSET downregulation was

12 accompanied by decreased levels of H3K36me2 in senescent MSCs. The osteogenic

13 potential of senescent MSCs greatly declined in parallel with MMSET downregulation.

14 Additionally, MMSET ablation effectively abolished the melatonin-enhanced osteogenic

15 differentiation of senescent MSCs. Thus, MMSET downregulation seems to be an

16 important characteristic of senescent MSCs, and it weakens the osteogenic differentiation

17 potential of MSCs. Given that MMSET is a histone methyltransferase, we speculate that

18 it affects osteogenic differentiation via epigenetic mechanisms. Ample evidences have

19 indicated that $\mathrm{H} 3 \mathrm{~K} 36 \mathrm{me} 2$ regulates transcription activity around the TSS region, such as

20 antagonizing Polycomb Repressive Complex 2 (PRC2)-mediated transcription silencing 
1 through $\mathrm{H} 3 \mathrm{~K} 27 \mathrm{me}$, and acts as a safeguard to ensure transcription fidelity(34).

2 Therefore, melatonin meliorates bone loss through enhancing transcription activities of

$3 \quad R U N X 2$ and SP7 mediated by MMSET, probably through modifying the histone

4 H3K36me2 around the TSS region of osteogenic gene promoter. Howerver, other

5 cis-elements or enhancer located far awy from the TSS regison should be considered. As

6 expected, MMSET overexpression led to an increase of H3K36me2 levels on the

7 promoters of osteogenic RUNX2 and SP7IOSTERIX genes before the induction of

8 osteogenesis. These results strongly indicate that MMSET may render the differentiation

9 propensity of MSCs toward osteoblasts via an epigenetic mechanism. Although our study

10 suggested MMSET is a critical responser to melatonin treatment in ameliorating the loss

11 of bone, it is probably only one part of the consequence of senescence and one that could

12 be of general relevance, since the loss of expression resulted in a general impact on loss

13 of bone. Other genes playing important roles in osteogenesis, or an integrated role under

14 melatonin stimulation should be considered.

15 Senile osteoporosis arises from multiple age related events associated with the aberrant

16 expression of multiple genes, and accumulating evidence links aging-associated

17 osteoporosis to epigenetic alterations(35). Epigenetic mechanisms are very important in

18 the acquisition of cell fate in stem cells. It is possible that aging triggers epigenetic

19 alterations, which bias the differentiation potential of bone marrow-derived MSCs. When

20 young well-differentiated cells enter into senescence, there is a drift in DNA methylation. 
1 A previous study revealed that the abundance of DNMT1 and DNMT3a decreases in

2 senescent cells, whereas DNMT3b expression increases steadily(26). In fact, DNA

3 methylation is well known for gene silencing of $B M P 2$, because higher $\mathrm{CpG}$ methylation

4 in the $B M P 2$ promoter is found in osteoporotic individuals compared with healthy

5 adults(36). Given that BMP2 is a pivotal molecule modulating bone formation, aberrant

6 methylation in its promoter region may result in impeded osteogenesis(36). Our research

7 indicates that the decrease of MMSET may be a consequence of aging due to DNA

8 methylation of the MMSET promoter. Analysis of the underlying mechanism showed that

9 the decrease of MMSET in senescent MSCs may be due to DNMT3b-mediated promoter

10 methylation, and treatment with melatonin effectively decreased the expression of

$11 D N M T 3 b$ to enhance MMSET expression. Dr. Reiter has proposed that melatonin exerts

12 DNMT inhibitory effects either by masking DNMT target sequences or by blocking the

13 active site of the enzyme(37). Our study investigation also suggests that melatonin

14 suppresses the expression of NDMT3b, thereby attenuates the DNA methylation status on

15 MMSET promoter, consequentially promotes the MMSET transcription activity. However,

16 the detailed working machinery of melatonin on DNMT3b suppression has not been

17 clarified in the current study and needs further investigation.

18 Taken together, our study identified MMSET as a modulator of melatonin-mediated

19 osteogenesis. Melatonin modifies the DNA methylation of the MMSET promoter in bone

20 marrow-derived MSCs to promote bone formation. Thus, this study highlights the 
1 importance of melatonin-MMSET pathway in aging-associated osteoporosis.

2 Nevertheless, the current study focuses on restoring and accentuating bone formation

3 using melatonin, but the in vivo bone dynamics comprise osteoblast-derived bone

4 formation and osteoclast-driven resorption(38). Thus, apart from the assessment of bone

5 formation in response to melatonin, the residual components of bone have not been

6 investigated in this study, although recent reports have revealed inhibitory effects of

7 melatonin on osteoclastogenesis $(39,40)$. In addition, melatonin has additional effects

8 unconducive to the treatment of age-related bone loss, whether it is amenable to

9 therapeutic purpose remains further investigation. 


\section{Data availability}

2 All data needed to evaluate the conclusions in the paper are present in the paper and/or

3 the Supplementary Materials. The ChIP-seq data was deposited at the Gene Expression

4 Omnibus database under accession number GSE158786. Requests for any materials in

5 this study should be directed to Zhiqiang Liu and obtained through an MTA.

6 Acknowledgement: All authors thank the reviewers for providing overarching

7 perspective in revision of this article, and we thank Professor Jun Qin at Shanghai

8 Institutes for Biological Sciences, Chinese Academy of Sciences for providing the NSD2

9 expressing plasmids and truncations. This work was supported by the National Natural

10 Science Foundation of China (81670201, 81870161, 82070221, Z.L.; 81900215, J.Y.

11 Wang; 81471175, X.L.), the Beijing Natural Science Foundation of China (Z200020), the

12 Natural Science Foundation of Tianjin (19JCZDJC35200, X.L.), the Talent Project of

13 Tianjin Medical University (11601501/2016KJ0317, Z.L.), the College Student

14 Innovation Program of Tianjin Medical University (201810062011, X.L.).

15 Authorship Contribution: All authors have read and approved the final manuscript.

16 Y.X., N.H., F.L., XY.B., XZ.L. contributed to the performance of in vitro experiments,

17 interpretation of data, and essential reagent or tools; S.W., JY.W., J.G., J.L., JJ.W., X.L.,

18 ML.H., CH.L. and HM.J. carried out animal experiments and interpretation of data; F.L.,

19 WX. L., ZJ.Z., H.Z. and LJ.W. provided clinical specimens; Y.X. and ZQ.L. contributed 
1 to the conception and design of the experiments, drafting of the manuscript, and critical

2 review of the manuscript.

3 Conflict-of-interest disclosure: The authors declare no competing financial interests. 


\section{References}

\section{Uncategorized References}

1. Pevet, P. (1998) [Melatonin and biological rhythms]. Therapie, 53, 411-420.

2. St Hilaire, M.A., Rahman, S.A., Gooley, J.J., Witt-Enderby, P.A. and Lockley, S.W. (2019) Relationship between melatonin and bone resorption rhythms in premenopausal women. Journal of bone and mineral metabolism, 37, 60-71.

3. Tordjman, S., Chokron, S., Delorme, R., Charrier, A., Bellissant, E., Jaafari, N. and Fougerou, C. (2017) Melatonin: Pharmacology, Functions and Therapeutic Benefits. Current neuropharmacology, 15, 434-443.

4. Majidinia, M., Reiter, R.J., Shakouri, S.K., Mohebbi, I., Rastegar, M., Kaviani, M., Darband, S.G., Jahanban-Esfahlan, R., Nabavi, S.M. and Yousefi, B. (2018) The multiple functions of melatonin in regenerative medicine. Ageing research reviews, 45, 33-52.

5. Lu, K.H., Lin, R.C., Yang, J.S., Yang, W.E., Reiter, R.J. and Yang, S.F. (2019) Molecular and Cellular Mechanisms of Melatonin in Osteosarcoma. Cells, 8.

6. Liu, J., Huang, F. and He, H.W. (2013) Melatonin effects on hard tissues: bone and tooth. International journal of molecular sciences, 14, 10063-10074.

7. Radio, N.M., Doctor, J.S. and Witt-Enderby, P.A. (2006) Melatonin enhances alkaline phosphatase activity in differentiating human adult mesenchymal stem cells grown in osteogenic medium via MT2 melatonin receptors and the MEK/ERK (1/2) signaling cascade. Journal of pineal research, 40, 332-342.

8. Li, T., Jiang, S., Lu, C., Yang, W., Yang, Z., Hu, W., Xin, Z. and Yang, Y. (2019) Melatonin: Another avenue for treating osteoporosis? Journal of pineal research, 66, e12548.

9. Armas, L.A. and Recker, R.R. (2012) Pathophysiology of osteoporosis: new mechanistic insights. Endocrinology and metabolism clinics of North America, 41, 475-486.

10. Halim, A., Ariyanti, A.D., Luo, Q. and Song, G. (2020) Recent Progress in Engineering Mesenchymal Stem Cell Differentiation. Stem cell reviews and reports.

11. Luchetti, F., Canonico, B., Bartolini, D., Arcangeletti, M., Ciffolilli, S., Murdolo, G., Piroddi, M., Papa, S., Reiter, R.J. and Galli, F. (2014) Melatonin regulates mesenchymal stem cell differentiation: a review. Journal of pineal research, 56, 382-397.

12. Wang, B., Wen, H., Smith, W., Hao, D., He, B. and Kong, L. (2019) Regulation effects of melatonin on bone marrow mesenchymal stem cell differentiation. J Cell Physiol, 234, 1008-1015.

13. Chu, Z.M., Li, H.B., Sun, S.X., Jiang, Y.C., Wang, B. and Dong, Y.F. (2017) Melatonin promotes osteoblast differentiation of bone marrow mesenchymal stem cells in aged rats. European review for medical and pharmacological sciences, 21, 4446-4456.

14. Han, Y., Kim, Y.M., Kim, H.S. and Lee, K.Y. (2017) Melatonin promotes osteoblast differentiation by regulating Osterix protein stability and expression. Scientific reports, 7, 5716.

15. Nakano, M., Ikegame, M., Igarashi-Migitaka, J., Maruyama, Y., Suzuki, N. and Hattori, A. (2019) Suppressive effect of melatonin on osteoclast function via osteocyte calcitonin. The Journal of endocrinology. 
16. Sharan, K., Lewis, K., Furukawa, T. and Yadav, V.K. (2017) Regulation of bone mass through pineal-derived melatonin-MT2 receptor pathway. Journal of pineal research, 63.

17. Chen, Q., Shou, P., Zheng, C., Jiang, M., Cao, G., Yang, Q., Cao, J., Xie, N., Velletri, T., Zhang, X. et al. (2016) Fate decision of mesenchymal stem cells: adipocytes or osteoblasts? Cell death and differentiation, 23, 1128-1139.

18. Hu, C. and Li, L. (2019) Melatonin plays critical role in mesenchymal stem cell-based regenerative medicine in vitro and in vivo. Stem cell research \& therapy, 10, 13.

19. Han, Y.S., Kim, S.M., Lee, J.H. and Lee, S.H. (2018) Co-Administration of Melatonin Effectively Enhances the Therapeutic Effects of Pioglitazone on Mesenchymal Stem Cells Undergoing Indoxyl Sulfate-Induced Senescence through Modulation of Cellular Prion Protein Expression. International journal of molecular sciences, 19.

20. Yoon, Y.M., Lee, J.H., Song, K.H., Noh, H. and Lee, S.H. (2020) Melatonin-stimulated exosomes enhance the regenerative potential of chronic kidney disease-derived mesenchymal stem/stromal cells via cellular prion proteins. Journal of pineal research, e12632.

21. Chen, W., Chen, X., Chen, A.C., Shi, Q., Pan, G., Pei, M., Yang, H., Liu, T. and He, F. (2019) Melatonin restores the osteoporosis-impaired osteogenic potential of bone marrow mesenchymal stem cells by preserving SIRT1-mediated intracellular antioxidant properties. Free radical biology \& medicine.

22. Rafat, A., Mohammadi Roushandeh, A., Alizadeh, A., Hashemi-Firouzi, N. and Golipoor, Z. (2019) Comparison of The Melatonin Preconditioning Efficacy between Bone Marrow and Adipose-Derived Mesenchymal Stem Cells. Cell journal, 20, 450-458.

23. Suofu, Y., Li, W., Jean-Alphonse, F.G., Jia, J., Khattar, N.K., Li, J., Baranov, S.V., Leronni, D., Mihalik, A.C., He, Y. et al. (2017) Dual role of mitochondria in producing melatonin and driving GPCR signaling to block cytochrome c release. Proc Natl Acad Sci U S A, 114, E7997-E8006.

24. Tan DX, R.R. (2019) Mitochondria: the birth place, battle ground and the site of melatonin metabolism cells. Melatonin Research, 2, 44-66.

25. Wagner, E.J. and Carpenter, P.B. (2012) Understanding the language of Lys36 methylation at histone H3. Nat Rev Mol Cell Biol, 13, 115-126.

26. Casillas, M.A., Jr., Lopatina, N., Andrews, L.G. and Tollefsbol, T.O. (2003) Transcriptional control of the DNA methyltransferases is altered in aging and neoplastically-transformed human fibroblasts. Molecular and cellular biochemistry, 252, 33-43.

27. Tan, D.X., Xu, B., Zhou, X. and Reiter, R.J. (2018) Pineal Calcification, Melatonin Production, Aging, Associated Health Consequences and Rejuvenation of the Pineal Gland. Molecules, 23.

28. Tan, D.X., Manchester, L.C., Reiter, R.J., Qi, W.B., Zhang, M., Weintraub, S.T., Cabrera, J., Sainz, R.M. and Mayo, J.C. (1999) Identification of highly elevated levels of melatonin in bone marrow: its origin and significance. Biochimica et biophysica acta, 1472, 206-214.

29. Zhang, W.L., Meng, H.Z., Yang, R.F., Yang, M.W., Sun, G.H., Liu, J.H., Shi, P.X., Liu, F. and Yang, B. (2016) Melatonin suppresses autophagy in type 2 diabetic osteoporosis. Oncotarget, 7, 52179-52194. 
30. Han, D., Huang, W., Li, X., Gao, L., Su, T., Li, X., Ma, S., Liu, T., Li, C., Chen, J. et al. (2016) Melatonin facilitates adipose-derived mesenchymal stem cells to repair the murine infarcted heart via the SIRT1 signaling pathway. Journal of pineal research, 60, 178-192.

31. Li, J., Li, N., Chen, Y., Hui, S., Fan, J., Ye, B., Fan, Z., Zhang, J., Zhao, R.C. and Zhuang, Q. (2019) SPRY4 is responsible for pathogenesis of adolescent idiopathic scoliosis by contributing to osteogenic differentiation and melatonin response of bone marrow-derived mesenchymal stem cells. Cell death \& disease, 10, 805.

32. Jauhari, A., Baranov, S.V., Suofu, Y., Kim, J., Singh, T., Yablonska, S., Li, F., Wang, X., Oberly, P., Minnigh, M.B. et al. (2020) Melatonin inhibits cytosolic mitochondrial DNA-induced neuroinflammatory signaling in accelerated aging and neurodegeneration. J Clin Invest, 130, 3124-3136.

33. Xie, Z. and Chng, W.J. (2014) MMSET: role and therapeutic opportunities in multiple myeloma. BioMed research international, 2014, 636514.

34. Huang, C. and Zhu, B. (2018) Roles of H3K36-specific histone methyltransferases in transcription: antagonizing silencing and safeguarding transcription fidelity. Biophys Rep, 4, 170-177.

35. Del Real, A., Riancho-Zarrabeitia, L. and Riancho, J.A. (2018) Epigenetic Aging in Osteoporosis. Journal of bone and mineral research : the official journal of the American Society for Bone and Mineral Research, 33, 1902-1903.

36. Raje, M.M. and Ashma, R. (2019) Epigenetic regulation of BMP2 gene in osteoporosis: a DNA methylation study. Molecular biology reports, 46, 1667-1674.

37. Korkmaz, A. and Reiter, R.J. (2008) Epigenetic regulation: a new research area for melatonin? J Pineal Res, 44, 41-44.

38. Datta, H.K., Ng, W.F., Walker, J.A., Tuck, S.P. and Varanasi, S.S. (2008) The cell biology of bone metabolism. J Clin Pathol, 61, 577-587.

39. Maria, S., Samsonraj, R.M., Munmun, F., Glas, J., Silvestros, M., Kotlarczyk, M.P., Rylands, R., Dudakovic, A., van Wijnen, A.J., Enderby, L.T. et al. (2018) Biological effects of melatonin on osteoblast/osteoclast cocultures, bone, and quality of life: Implications of a role for MT2 melatonin receptors, MEK1/2, and MEK5 in melatonin-mediated osteoblastogenesis. J Pineal Res, 64.

40. Nakano, M., Ikegame, M., Igarashi-Migitaka, J., Maruyama, Y., Suzuki, N. and Hattori, A. (2019) Suppressive effect of melatonin on osteoclast function via osteocyte calcitonin. J Endocrinol, 242, 13-23. 


\section{Figure legends}

a

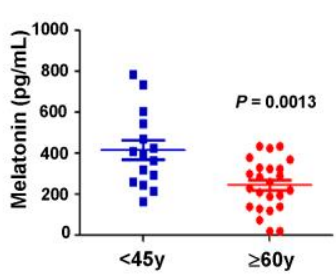

d

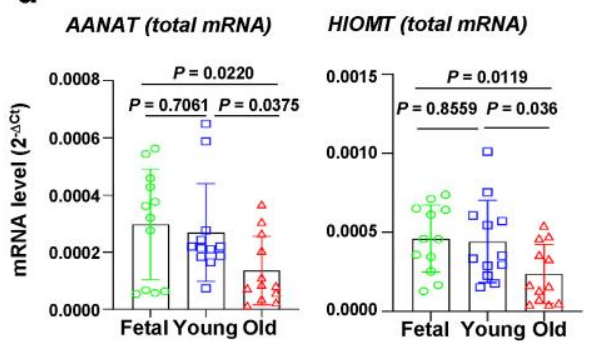

f
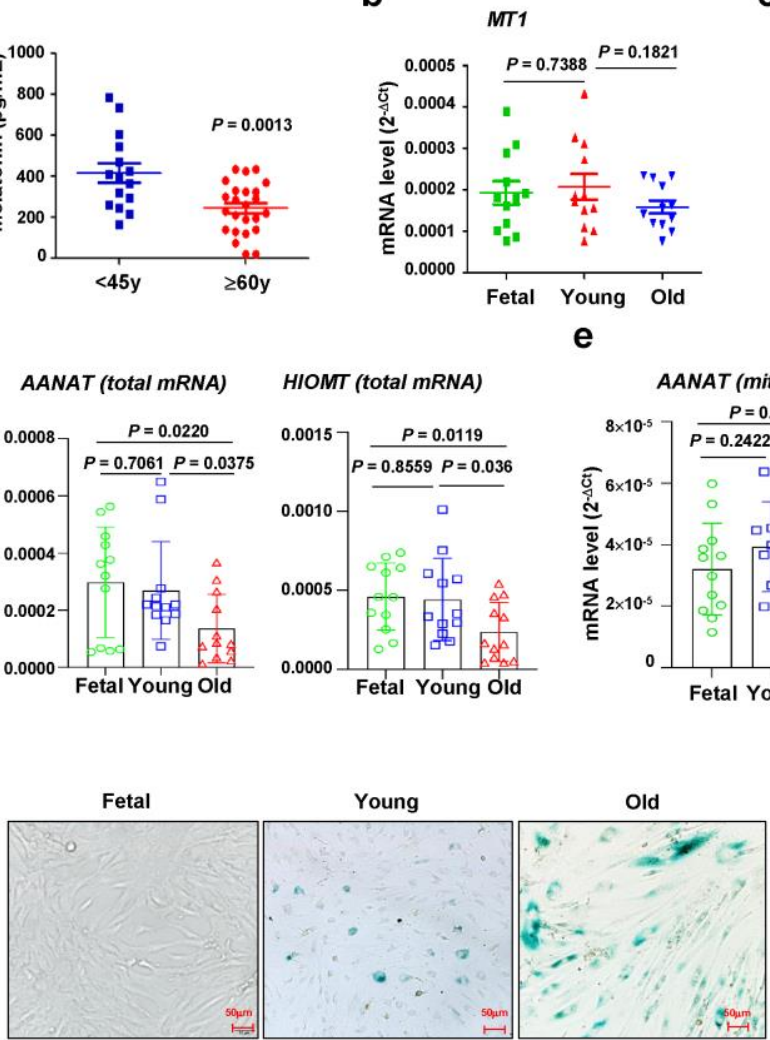

e
2 b

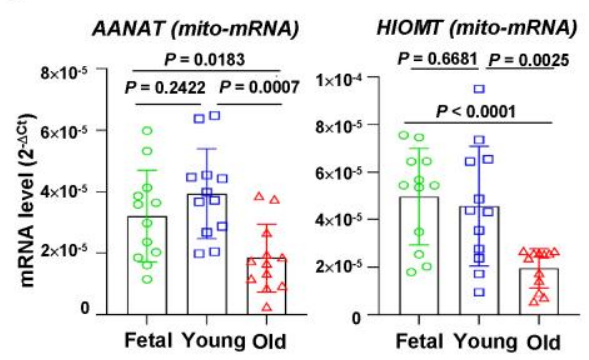

g

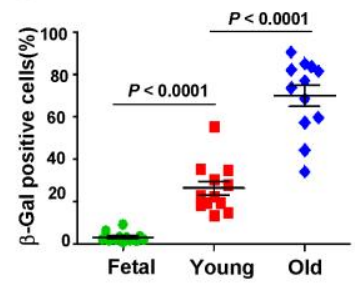

Figure 1

FIGURE 1 Melatonin in bone marrow decreases with aging. (a) Melatonin levels in

the bone marrow plasma of donors under 45 years old $(n=15)$ or over 60 years old $(n=24)$

measured by ELISA. mRNA levels of MT1 (b), MT2 (c), AANATand HIOMT (d) in total

cell lysate (e) or mitochondrion of bone marrow MSCs derived from aborted fetuses (<

22 weeks, $n=12)$, young adults $(16-45$ years, $n=12)$, and the old (> 60 years, $n=12)$. n.s.,

no significance. (f) Representative images of activity of lysosomal $\beta$-galactosidase in

MSCs from donors with different ages. Scale bar, $50 \mu \mathrm{m}$. (g) Quantification of the

percentage of $\beta$-galactosidase staining positive MSCs in three groups $(n=12)$. For the 
1 ELISA assay, each sample was triplicated; for the quantification of $\beta$-galactosidase

2 staining, 9 random vision field with $200 \times$ magnification were analyzed.

a

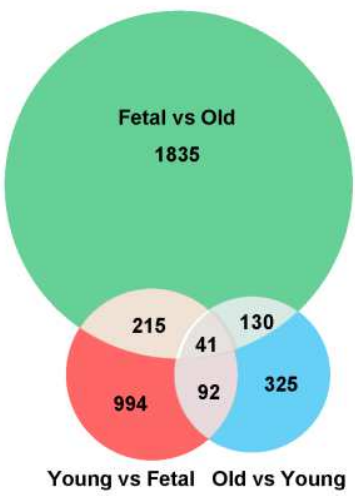

C

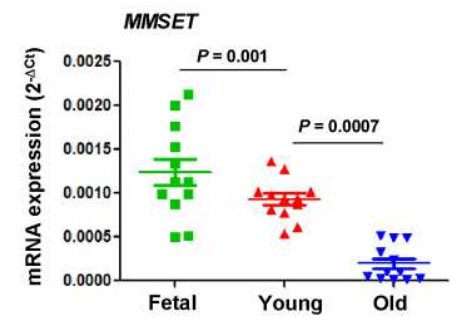

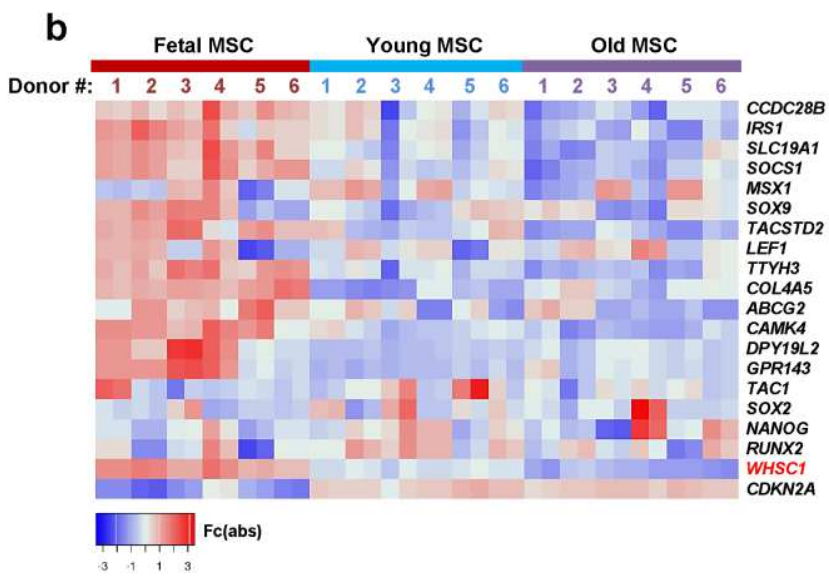

d

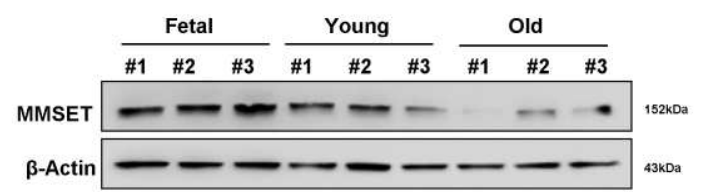

Figure 2

4 FIGURE 2 Gene expression profile assay identifies MMSET downregulation in

5 senescent MSCs. (a) Venn diagram shows the number of differently expressed genes in

6 three comparisons of three groups. (b) Heat map shows hierarchical clustering of altered

7 genes in MSCs derived from donors of different ages. Fc, fold change; cut off: $\mathrm{Fc}>2$ and

$8 \quad P<0.01$. (c) mRNA levels of MMSET in MSCs derived from donors of different ages (n

$9=12$ with each detection triplicated). (d) Representative protein levels of MMSET in

10 MSCs from the three groups. All Western blot results represents a representative example

11 of three independently performed studies, each yielding similar results. 
a
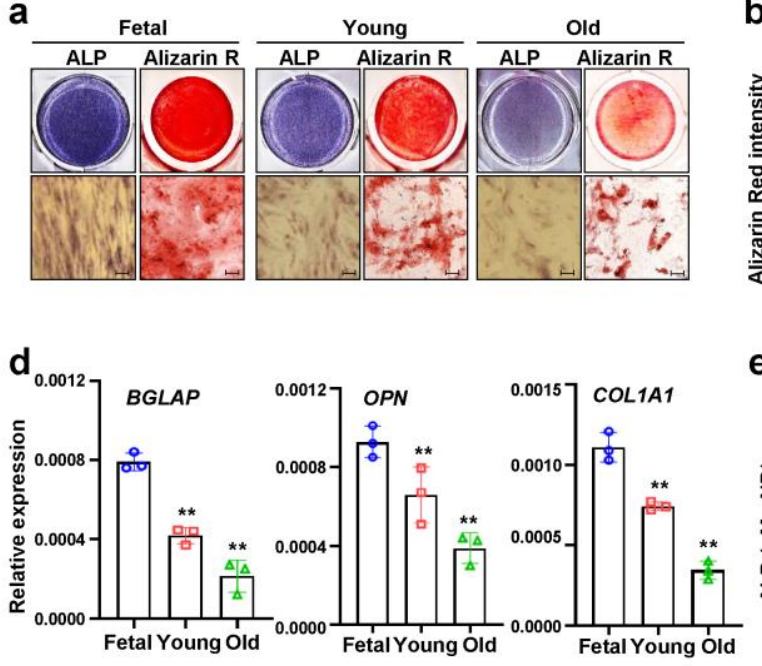

g

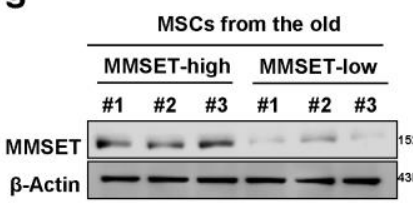

h

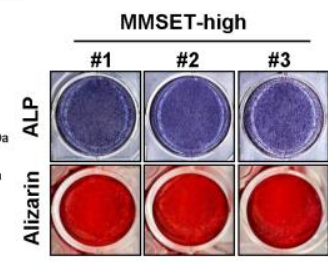

b

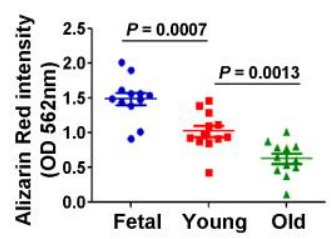

e $f$

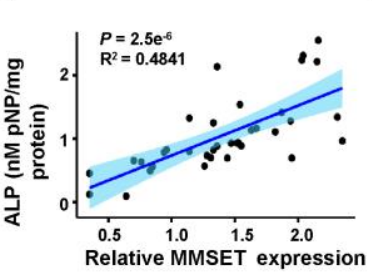

C

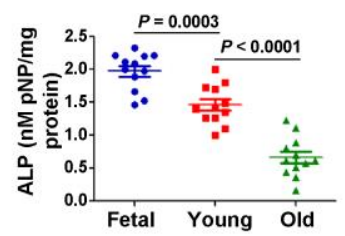

f

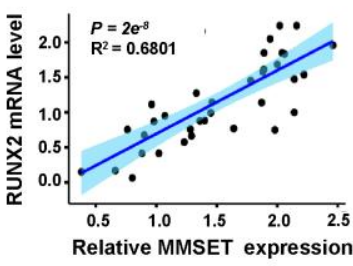

2

Figure 3

i

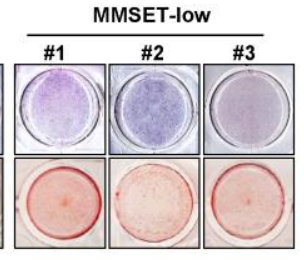

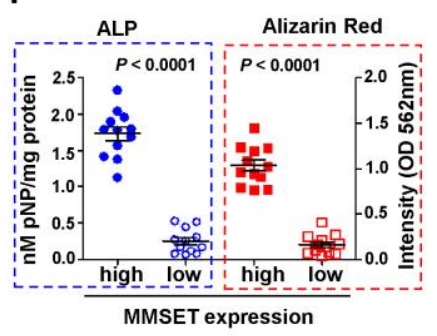

\section{FIGURE 3 Osteogenic potential of senescent MSCs declines in parallel to MMSET}

downregulation. (a) Representative images of alkaline phosphatase assay (ALP) staining and Alizarin Red S assay for MSCs from fetal, young or old donors cultured with osteogenic media for 14 days. Scale bar, $100 \mu \mathrm{m}$. (b) and (c) Quantification of Alizarin Red S staining and ALP staining of 12 donors in each group $(n=12$ with each detection triplicated). (d) Osteogenic marker genes expressions in MSCs from different groups cultured with osteogenic media for 14 days (n=3 with each detection triplicated). ${ }^{* *}, P<$ 0.01. (e) Correlation of ALP levels and MMSET expression in MSCs of different groups (n = 36). (f) Correlation of RUNX2 and MMSET expression in MSCs of different age 
1 groups $(\mathrm{n}=36) .(\mathbf{g})$, Representative of MMSET protein level in senescent MSCs $(\mathrm{n}=12)$.

2 (h) Representative images of ALP staining and Alizarin Red S assay in senescent MSCs

3 with different MMSET levels. (i) Quantification of ALP staining and Alizarin Red

4 staining ( $\mathrm{n}=12$ with each sample triplic). All Western blot results represents a

5 representative example of three independently performed studies, each yielding similar

6 results.

a

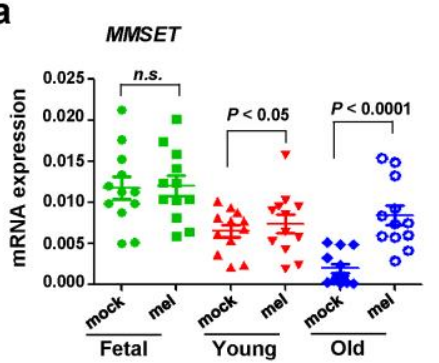

d
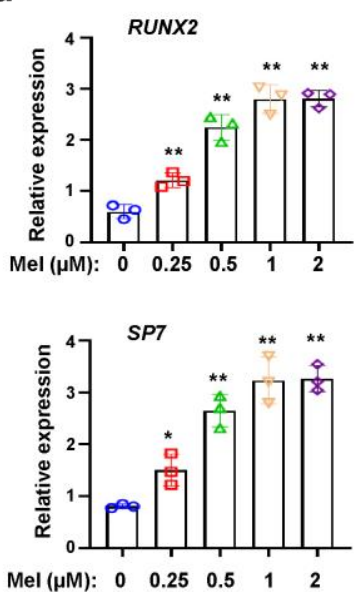

7 b

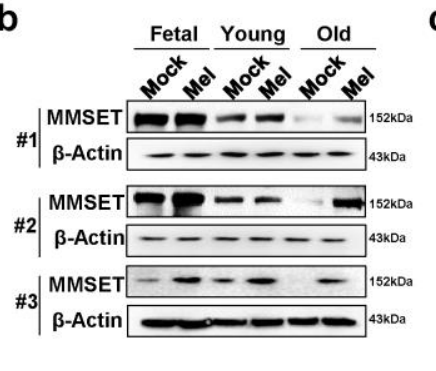

e

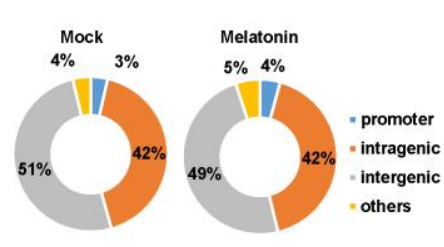

g

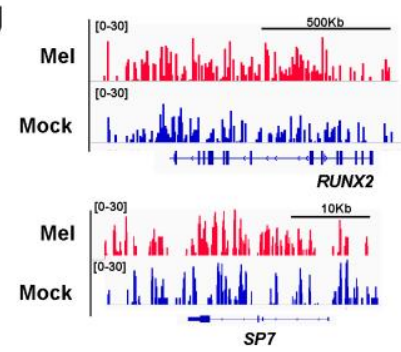

C

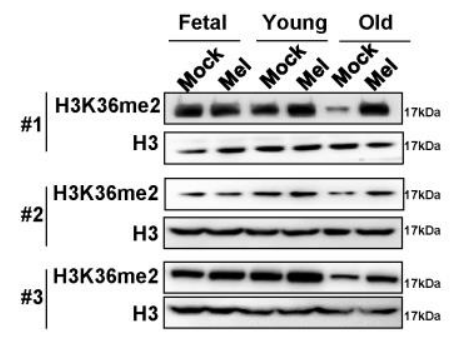

f

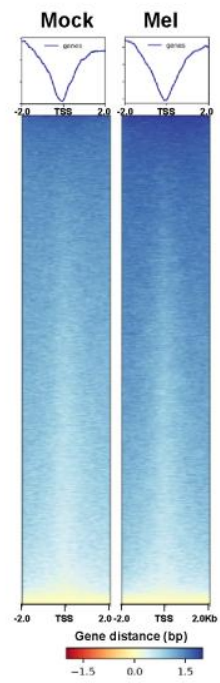

Figure 4

FIGURE 4 Melatonin stimulates MMSET expression in senescent MSCs. (a) mRNA level of MMSET of MSCs from donors of different ages treated with melatonin ( $\mathrm{n}$ $=12$ with each sample triplicated). n.s., no significance. (b) The MMSET expression in three primary MSCs from donors of different ages treated with melatonin. (c) The 
1 H3K36me2 levels in three primary MSCs from donors of different ages treated with

2 melatonin. (d) mRNA levels of RUNX2 and SP7/OSTERIX in MSCs treated with

3 increasing dosage of melatonin for 24 hours $\left(\mathrm{n}=3\right.$ with each detection triplicated). ${ }^{*}, P<$

$4 \quad 0.05$; **, $P<0.01$. (e) Pie chart for ChIP-seq profile to show percentage of genes

5 enriched by H3K36me2 in MSC cells treated with vehicle or $1 \mu \mathrm{M}$ melatonin $(\mathrm{n}=3)$; (f)

6 Changes of H3K36me2 density around the TSS region of genes. (g) Representative

7 ChIP-seq profiles for $\mathrm{H} 3 \mathrm{~K} 36 \mathrm{me} 2$ occupancy at the promoter regions of $R U N X 2$ and

8 SP7/OSTERIX genes. All Western blot results represents a representative example of

9 three independently performed studies, each yielding similar results.
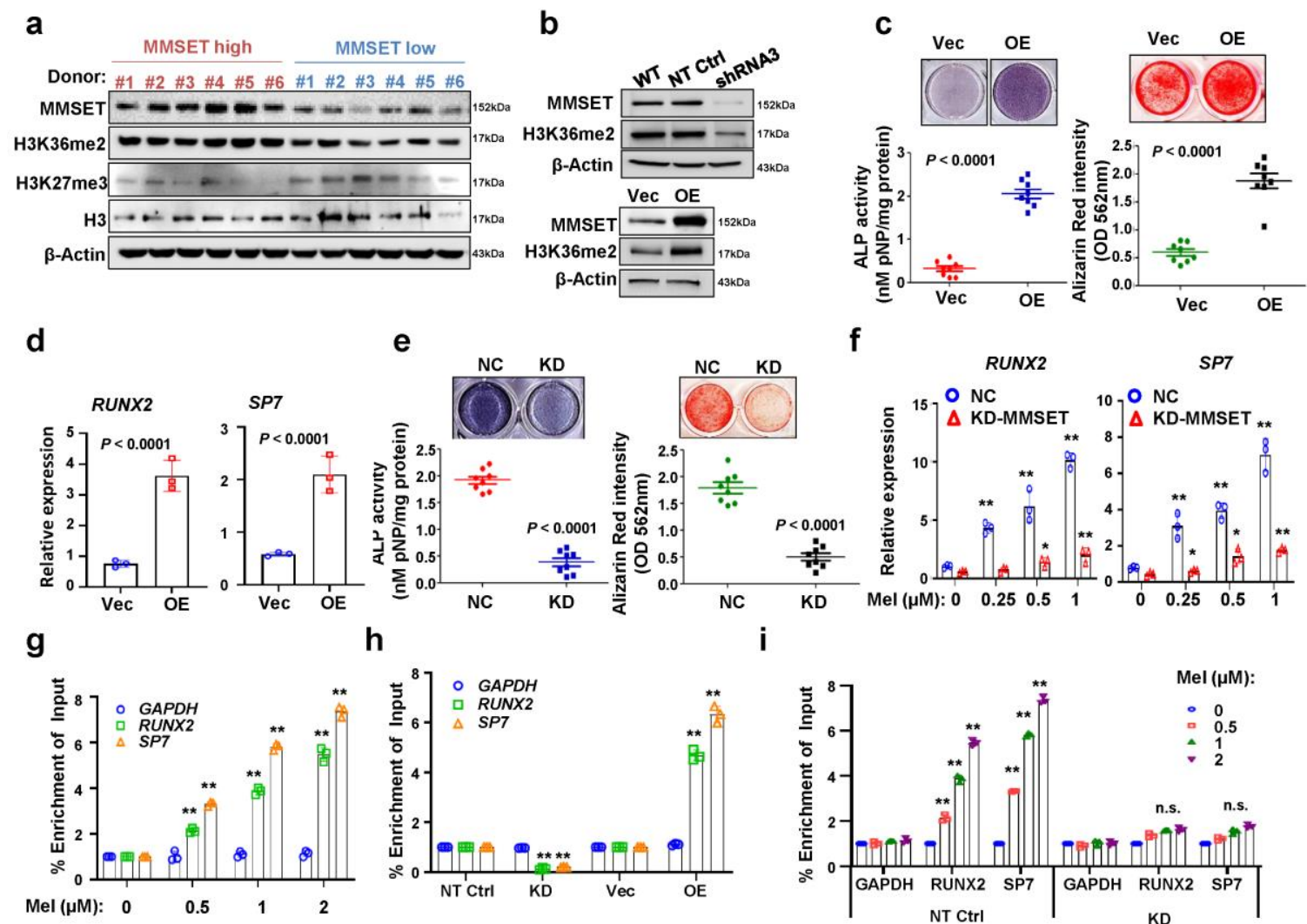

h

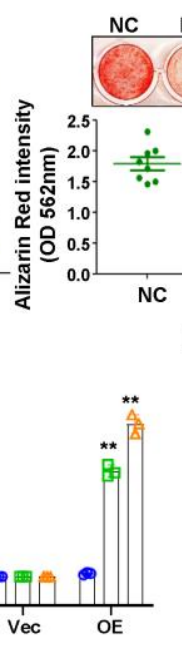

f
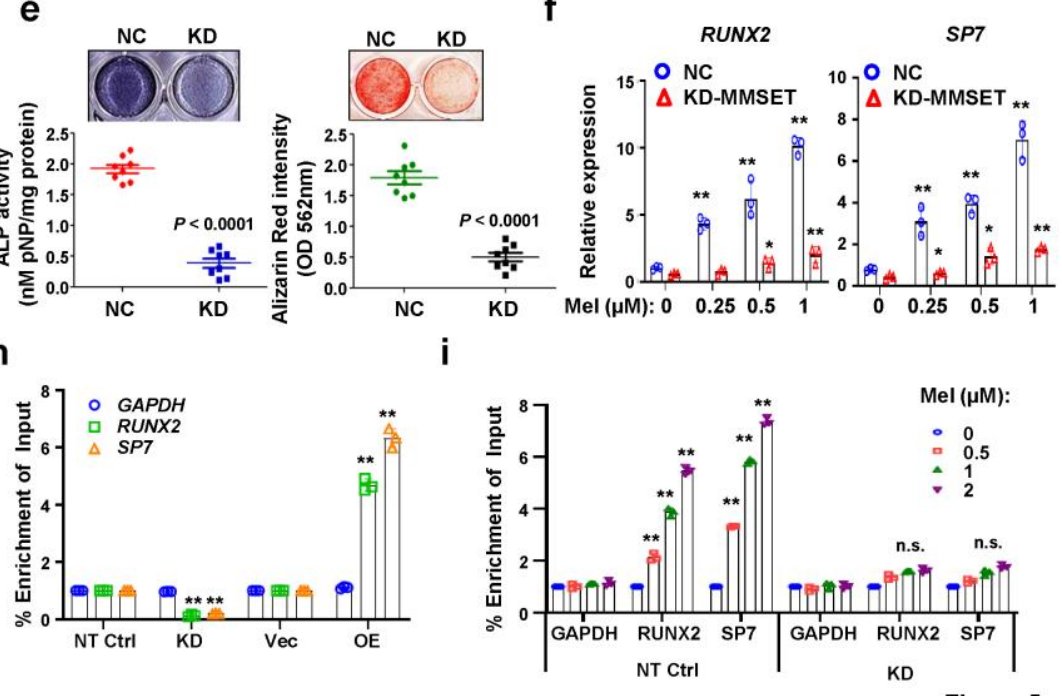

Figure 5 
1 FIGURE 5 MMSET favors osteogenic differentiation. (a) Levels of MMSET,

2 H3K36me2 and H3K27me3 in MSCs (n=6). (b) Expression of MMSET and the

3 corresponding H3K36me2 level in MSCs with MMSET knockdown (upper panel) or

4 overexpression (lower panel). (c) The representative images (upper panel) and

5 quantification (lower panel) of ALP and Alizarin Red S assay in MSCs with MMSET

6 overexpression $(\mathrm{n}=8)$. (d) Expressions of RUNX2 and SP7/OSTERIX in MSCs with

7 MMSET overexpression ( $\mathrm{n}=3$ with each detection triplicated). (e) The representative

8 images (upper panel) and quantification (lower panel) of ALP and Alizarin Red S assay in

9 MSCs with MMSET silencing $(\mathrm{n}=8)$. (f) RUNX2 and SP7/OSTERIX expressions in

10 MSCs with MMSET silencing treated with increasing amount of melatonin for 24 hours

11 (n = 3 with each detection triplicated). *, $P<0.05 ; * *, P<0.01 . \mathbf{g}$, h and $\mathbf{i}$, ChIP-qPCR

12 analysis showed the recruitment of H3K36me2 on RUNX2 and SP7 promoter in MSCs

13 treated with increasing dosage of melatonin for 24 hours (g), with overexpressed

14 MMSET (h), or with MMSET silencing and then treated with increasing dosage of

15 melatonin for 24 hours (i). The values represent mean \pm standard error of the mean (SEM)

16 ( $\mathrm{n}=3$ with each detection triplicated). n.s., no significance. $* *, P<0.01$. All Western blot

17 results represents a representative example of three independently performed studies, 18 each yielding similar results. 


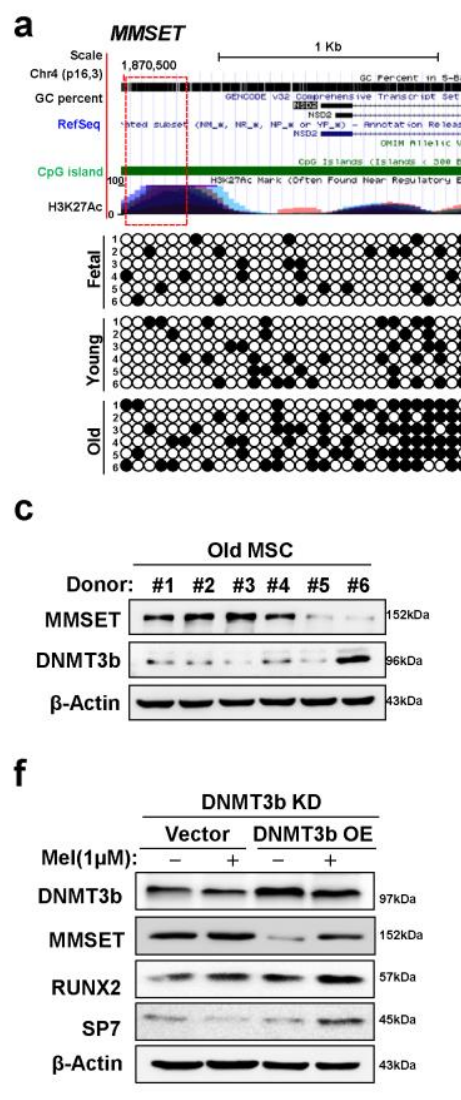

b
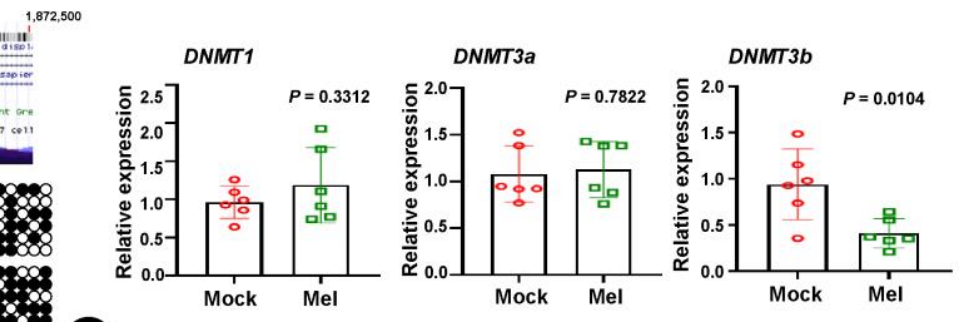

d

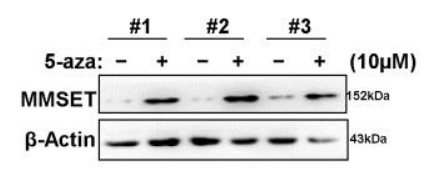

g

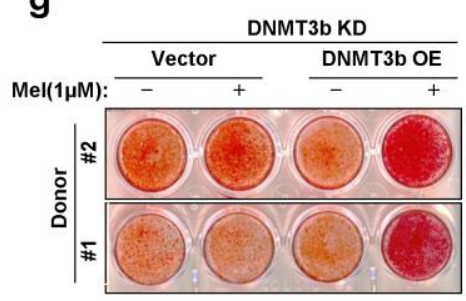

e

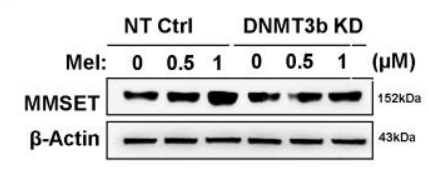

h

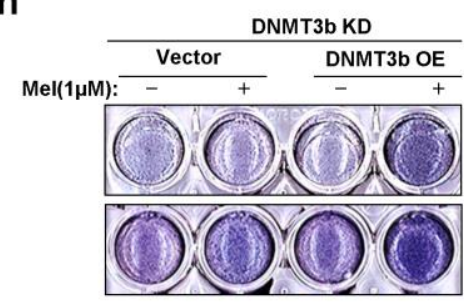

Figure 6

2 FIGURE 6 Melatonin alleviates DNA methylation of MMSET promoter. (a)

3 Methylation signatures at an island on MMSET gene promoter in MSCs from different

4 ages groups. (b) Expressions of DNMTs of senescent MSCs treated with $1 \mu \mathrm{M}$ melatonin

5 for 24 hours determined by real time PCR $(n=6)$. (c) Expressions of MMSET and

6 DNMT3b in senescent MSCs $(\mathrm{n}=6)$. (d) MMSET expression in 3 senescent MSCs

7 treated with $10 \mu \mathrm{M}$ 5-aza. (e) Expression of MMSET in MSCs with DNMT3b

8 knockdown (KD) and treated with increasing dosage of melatonin for 24 hours. (f)

9 DNMT3b, MMSET, RUNX2 and SP7 expression in the DNMT3b-knockdown MSCs

10 with DNMT3b overexpression and treated with vehicle or $1 \mu \mathrm{M}$ melatonin.

11 Representative images of Alizarin Red S assay (g) and ALP staining (h) in the 
1 DNMT3b-knockdown MSCs with DNMT3b overexpression and treated with vehicle or 1

$2 \mu \mathrm{M}$ melatonin cultured with osteogenic media for 14 days. All Western blot results

3 represents a representative example of three independently performed studies, each

4 yielding similar results.
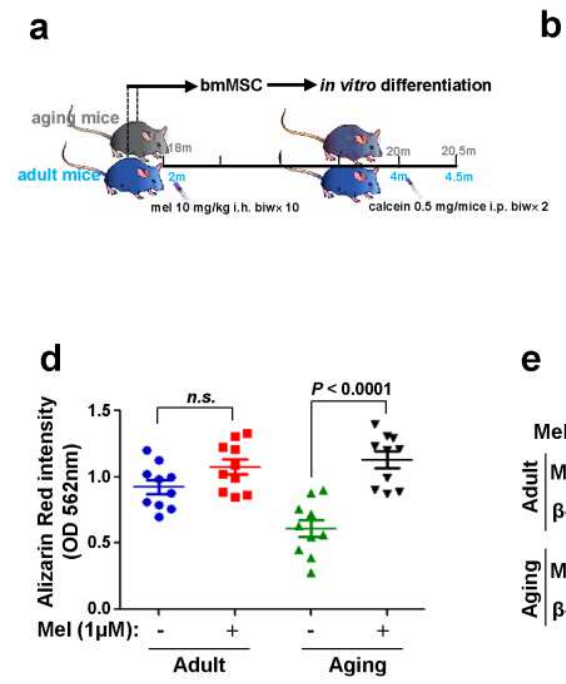

g

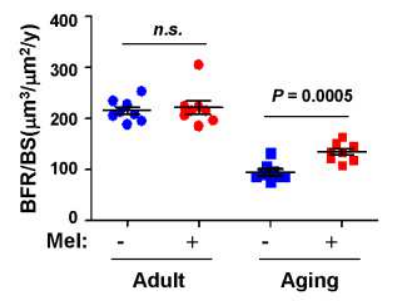

b

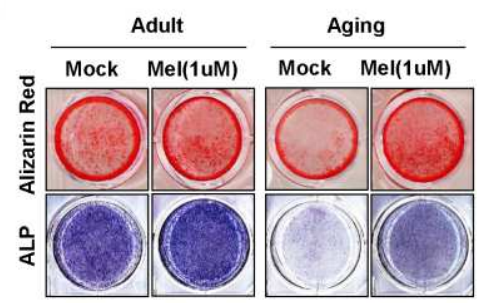

e

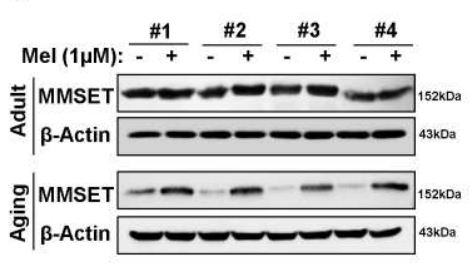

h

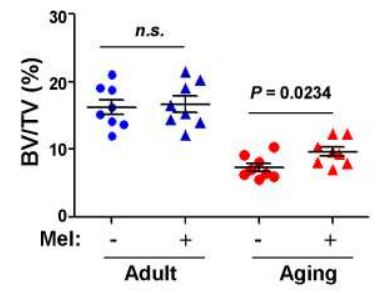

C

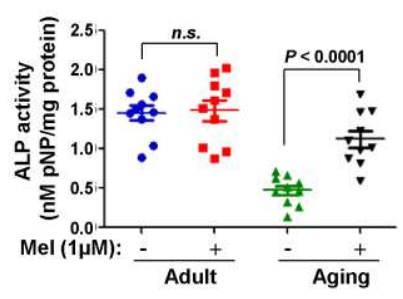

f

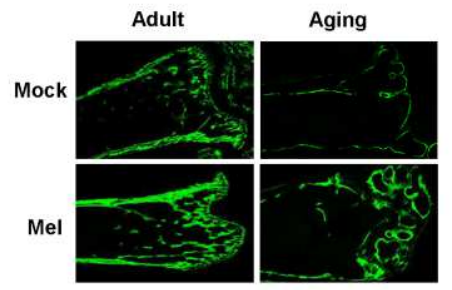

i

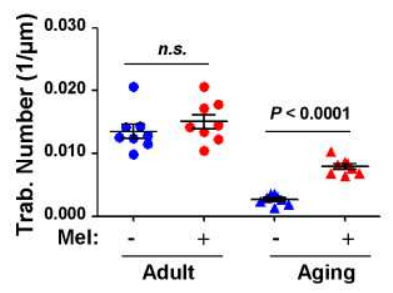

5

FIGURE 7 Melatonin facilitates osteogenesis of MSCs from aging mice via MMSET. (a) Treatment schedule and experiment design in aging mice and adult mice. (b) Representative images of ALP and Alizarin Red S staining of MSCs cultured with osteogenic media for 14 days from aging and young mice in presence with vehicle or melatonin. Quantification of ALP (c) and Alizarin Red S staining (d) of MSCs from aging 
1 and young mice groups treated with vehicle or melatonin $(\mathrm{n}=10)$. (e) Representative

2 MMSET expression in MSCs from aging and young mice treated with vehicle or

3 melatonin for 24 hours. (f) The representative images of calcein stained femur bone from

4 aging and young mice treated with vehicle or melatonin for 10 weeks. Quantification of

5 bone formation rate (BFR/BS) (g), the percentage of bone volume to total volume

6 (BV/TV) (h) and number of bone trabecula (i) in femurs of aging and young mice treated

7 with vehicle or melatonin $(n=8)$. n.s., no significance. All Western blot results represents

8 a representative example of three independently performed studies, each yielding similar

9 results.

a

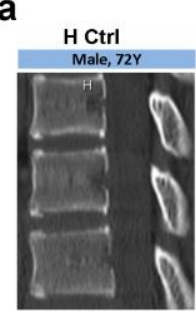

d

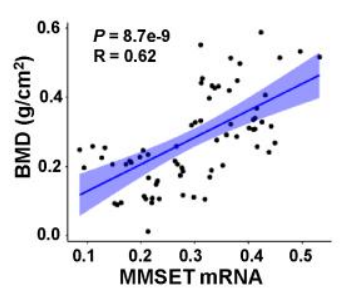

g

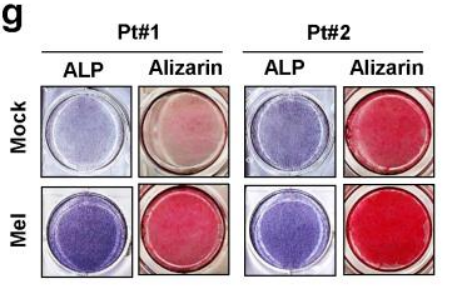

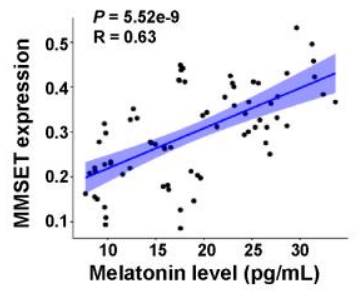

b c

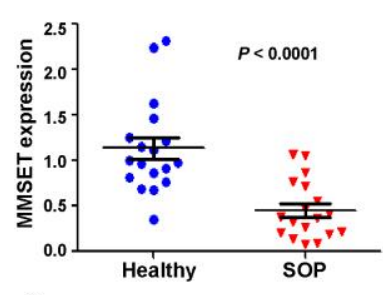

f

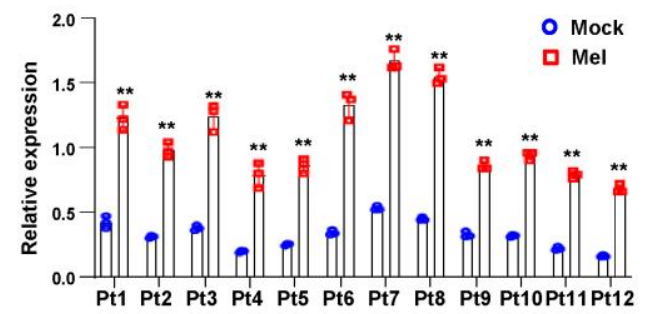

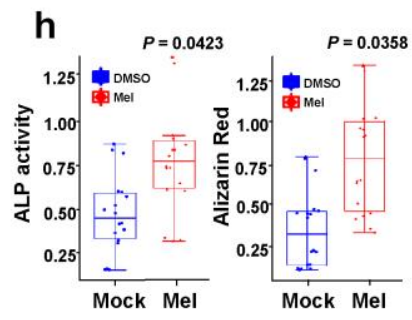


1 FIGURE 8 Melatonin treatment recovers osteogenic potential of MSCs from

2 patients with senile osteoporosis. (a) Radiographic images of healthy control (H Ctrl)

3 and patients with senile osteoporosis $(\mathrm{Pt})$. Red arrows indicate the typical area with

4 osteoporosis. (b) MMSET expression in MSCs isolated from healthy control and patients

5 with senile osteoporosis ( $\mathrm{n}=18$ with each detection triplicated). Correlation of bone mass

6 density (BMD) with melatonin level in bone marrow plasma (c), and MMSET expression

7 in MSCs from senile osteoporosis patients (d) (3 independent reads for $n=12$ patients).

8 (e) Correlation of MMSET expression in MSCs and melatonin level in bone marrow

9 plasma of patients with senile osteoporosis (3 independent reads for $n=12$ patients). (f)

10 MMSET expression in 12 MSCs from patients with senile osteoporosis treated with $1 \mu \mathrm{M}$

11 melatonin for 24 hours $(\mathrm{n}=3$ with each detection triplicated). $* *, P<0.01$. (g) The

12 representative images of ALP and Alizarin Red S staining in MSCs from donors with

13 senile osteoporosis treated with osteogenic media for 14 days in presence of DMSO

14 vehicle or melatonin. (h) Quantification of ALP and Alizarin Red S staining for MSCs

15 under osteogenesis induction and in presence of DMSO vehicle or melatonin (3

16 independent reads for $\mathrm{n}=6 \mathrm{MSCs}$ ). (i) mRNA levels of RUNX2 and SP7/OSTERIX in

17 MSCs under osteogenesis induction and in presence of DMSO vehicle or melatonin $(\mathrm{n}=$

183 with each detection triplicated). $* *, P<0.01$. 

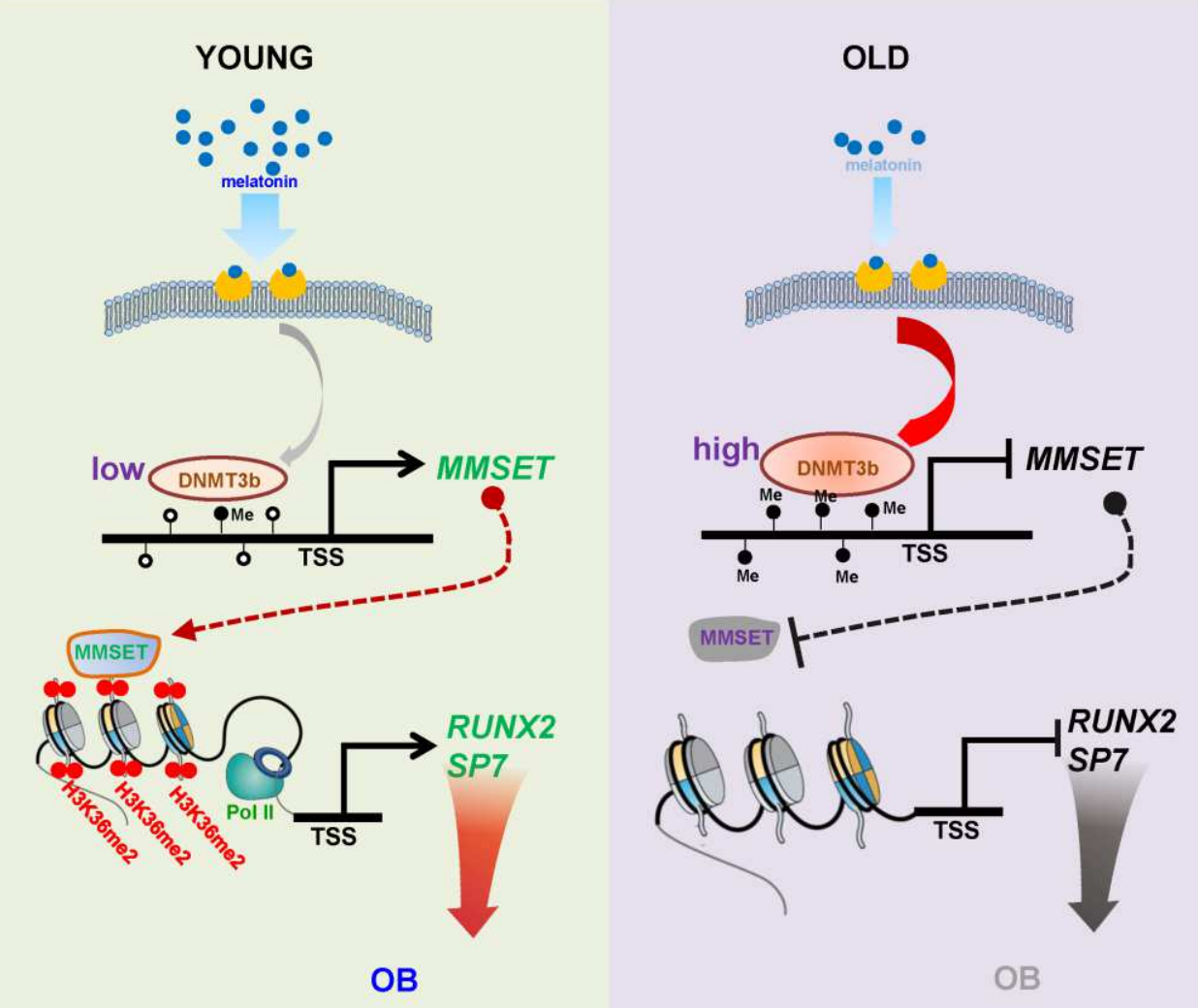

Figure 9

2 FIGURE 9 Proposed schematic diagram for mechanisms of promoting

3 osteoblastogenesis from senescent BMSCs by melatonin: melatonin alleviates the

4 senescence-related hypermethylation of the MMSET promoter, upregulates expression of

5 the histone methyltransferase NSD2; NSD2 promotes the histone H3 dimethylation

6 modification at lysine 36 of the osteogenic genes RUNX2 and SP7/OSTERIX as a result,

7 and consequentially enhances osteoblastogenesis from BMSCs. 
a

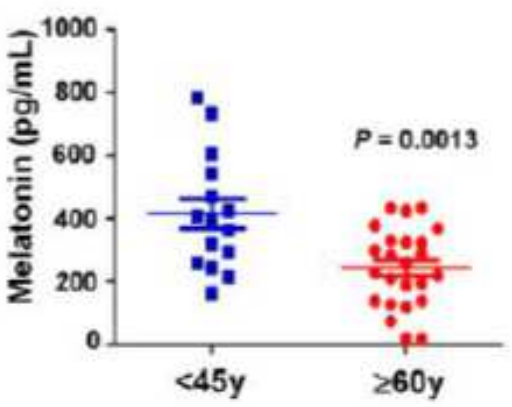

d

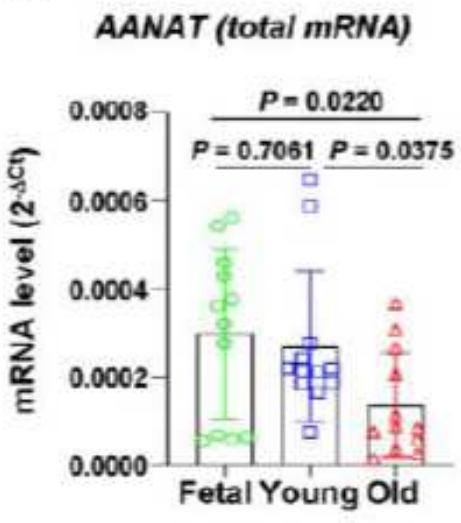

f

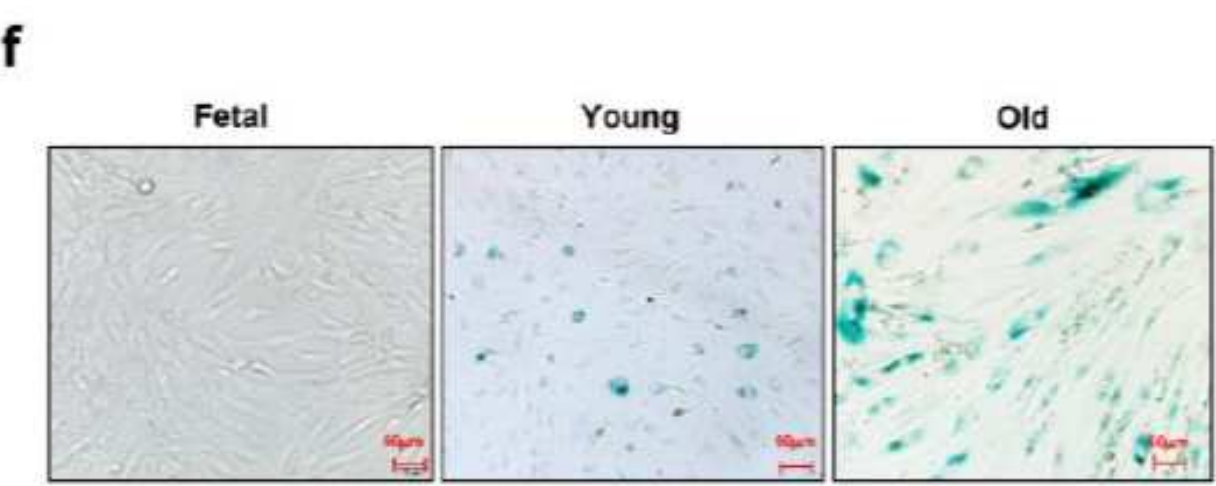

b

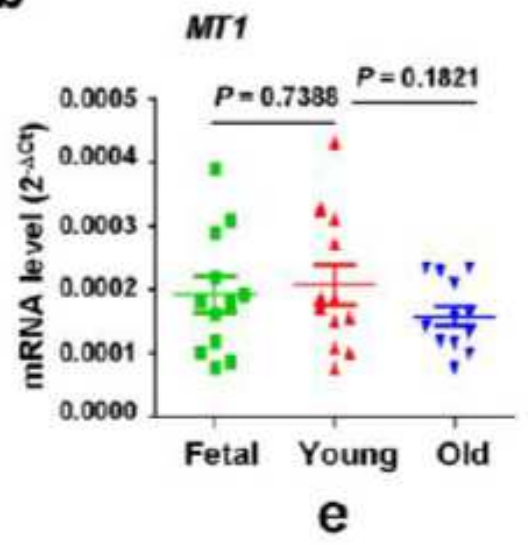

C

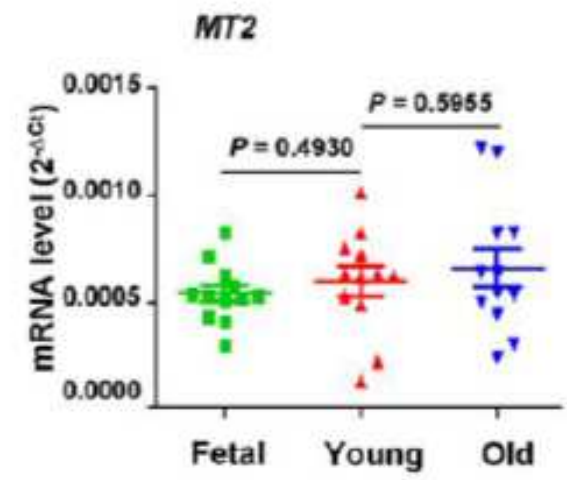

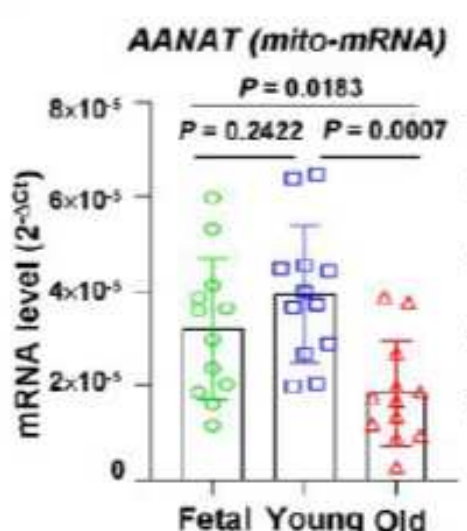

Fetal Young OId
HIOMT (mito-mRNA)

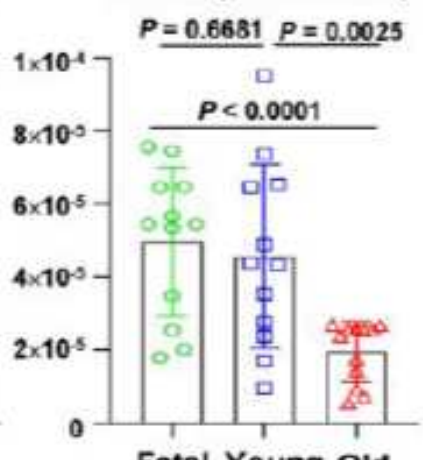

Fetal Young Old

g

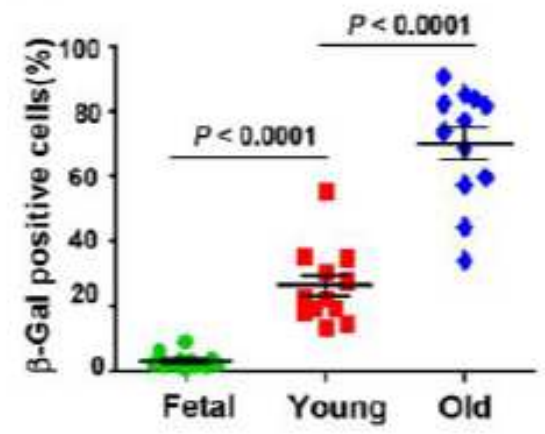

\section{Figure 1}

Melatonin in bone marrow decreases with aging. (a) Melatonin levels in the bone marrow plasma of donors under 45 years old $(n=15)$ or over 60 years old $(n=24)$ measured by ELISA. mRNA levels of MT1 (b), MT2 (c), AANATand HIOMT (d) in total cell lysate (e) or mitochondrion of bone marrow MSCs derived from aborted fetuses ( $<22$ weeks, $n=12$ ), young adults ( $16-45$ years, $n=12)$, and the old ( $>60$ years, $n=12$ ). n.s., no significance. (f) Representative images of activity of lysosomal $\beta$-galactosidase in MSCs from donors with different ages. Scale bar, $50 \mu \mathrm{m}$. (g) Quantification of the percentage of $\beta$-galactosidase 
staining positive MSCs in three groups ( $n=12)$. For the ELISA assay, each sample was triplicated; for the quantification of $\beta$-galactosidase staining, 9 random vision field with 200x magnification were analyzed.

a

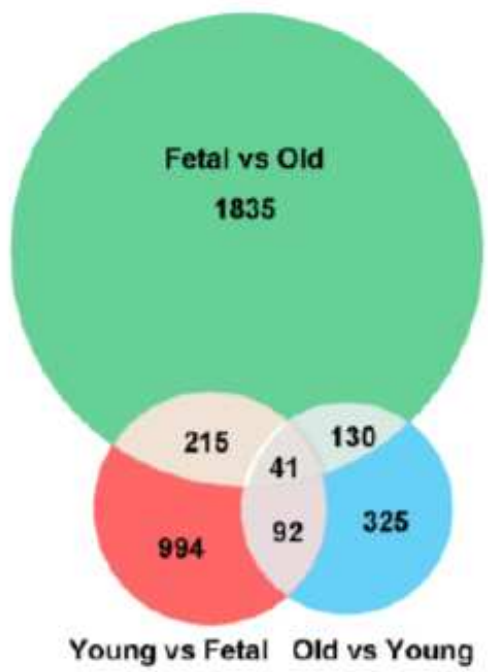

C

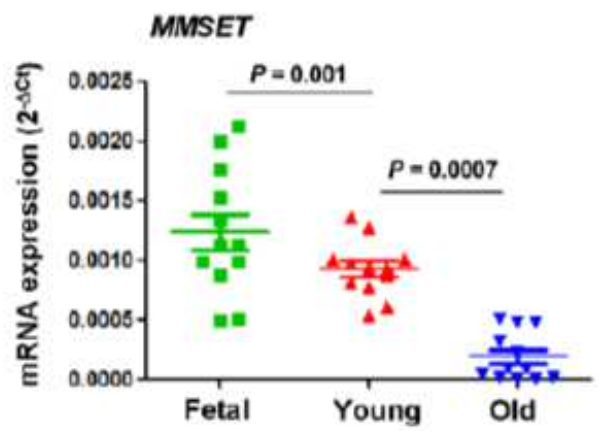

b

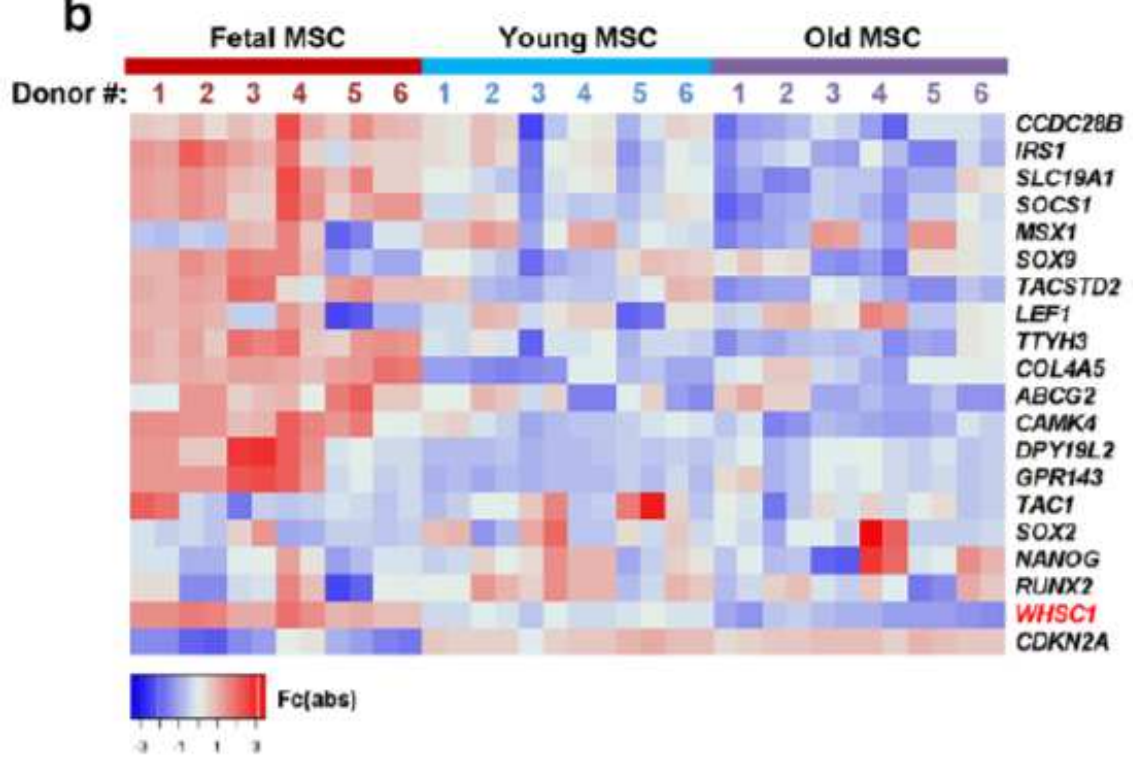

d

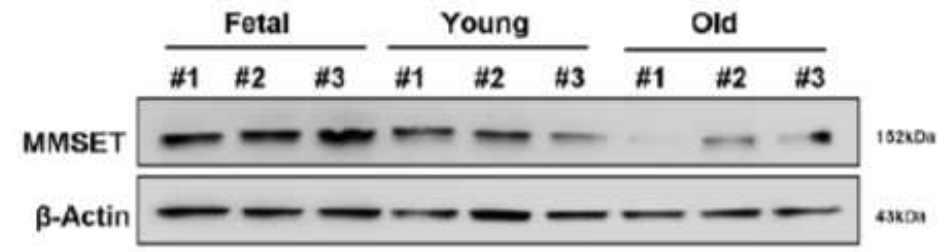

\section{Figure 2}

Gene expression profile assay identifies MMSET downregulation in senescent MSCs. (a) Venn diagram show s the number of differently expressed genes in three comparisons of three groups groups. (b) Heat map shows hierarchical clustering of altered genes in MSCs derived from donors of different ages ages. $F c$, fold change; cut off: $F c>2$ and $P<0.01$ 0.01. (c) mRNA levels of MMSET in MSCs derived from donors of different ages ( $n=12$ with each detection triplicated triplicated). (d) Representative protein levels of MMSET in MSCs from the three groups groups. All Western blot results represents a representative example of three independently performed studies, each yielding similar results. 
a
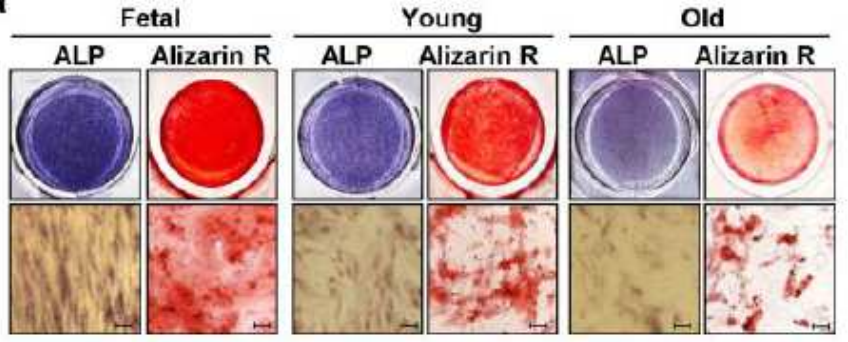

b

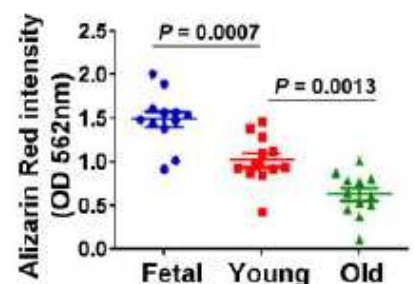

e

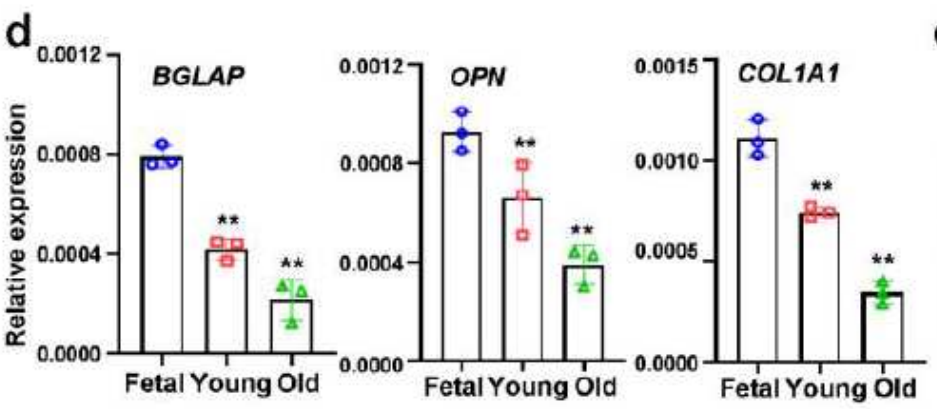

g

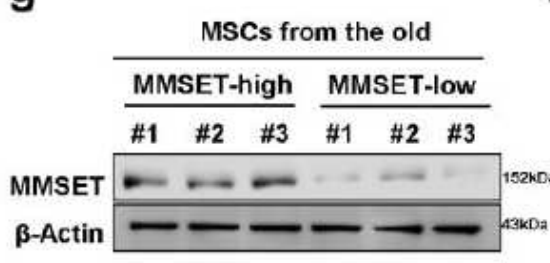

h

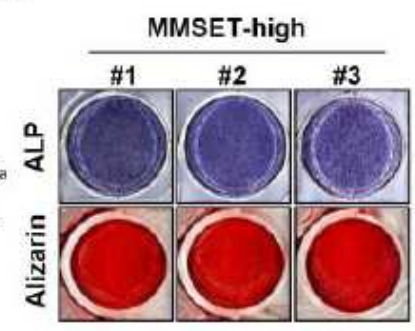

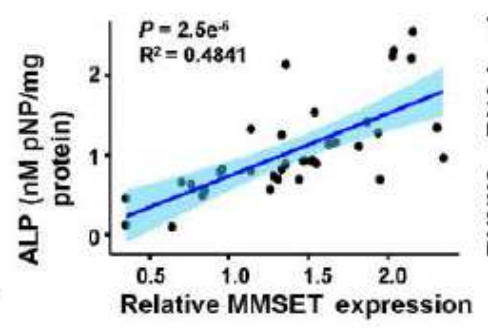

i

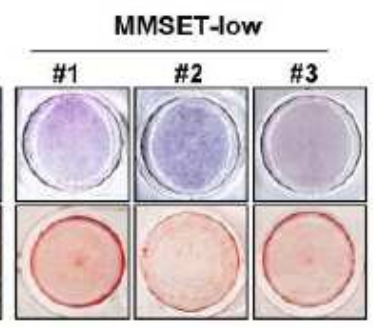

f

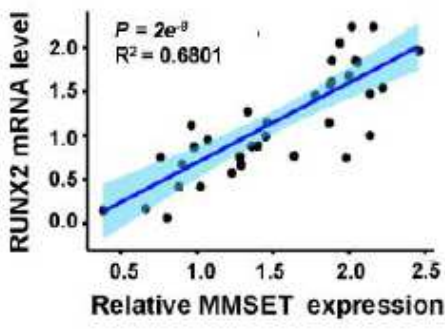

C
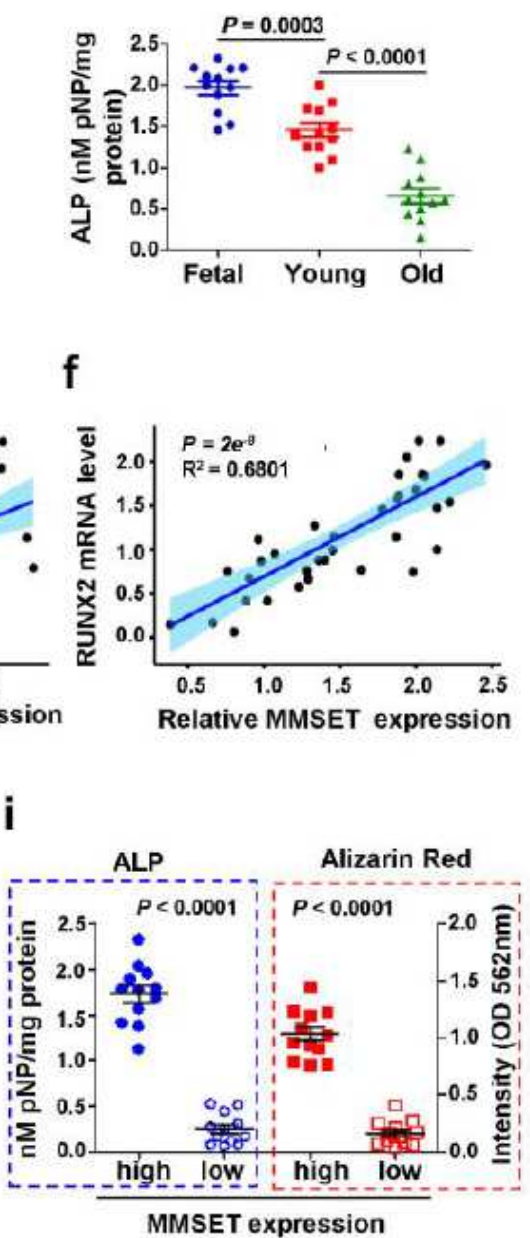

Figure 3

Osteogenic potential of senescent MSCs declines in parallel to MMSET downregulation. (a) Representative images of alkaline phosphatase assay (ALP) staining and Alizarin Red S assay for MSCs from fetal, young or old donors cultured with osteogenic media for 14 days. Scale bar, $100 \mu \mathrm{m}$. (b) and c) Quantification of Alizarin Red S staining and ALP staining of 12 donors in each group ( $\mathrm{n}=12$ with each detection triplicated). (d) Osteogenic marker gene genes expressions in MSCs from different groups cultured with osteogenic media for 14 days ( $n=3$ with each detection triplicated). ${ }^{*}, P<0.01$. (e) Correlation of ALP levels and MMSET expression in MSCs of different groups $(n=36)$. ( $f)$ Correlation of RUNX2 and MMSET expression in MSCs of different age groups $(n=36)$. $(g)$, Representative of MMSET protein level in senescent MSCs $(n=12)$. (h)Representative images of ALP staining and Alizarin Red $S$ assay in senescent MSCs with different MMSET levels. (i) Quantification of ALP staining and Alizarin Red staining ( $n=12$ with each sample triplic). All Western blot results represents a representative example of three independently performed studies, each yielding similar results. 
a

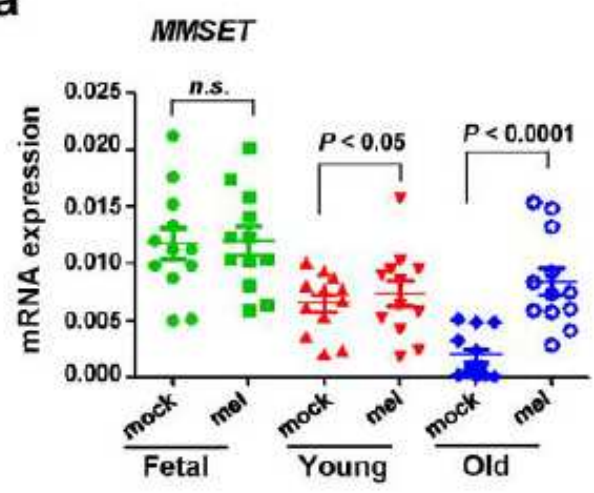

d
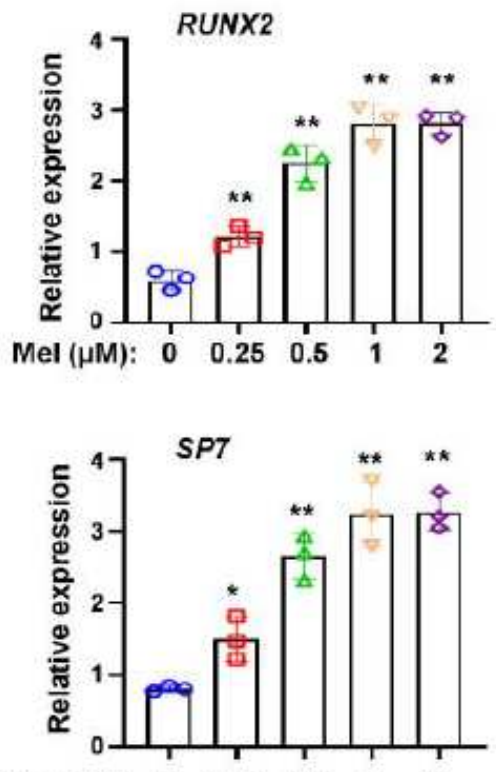

Mel $(\mu \mathrm{M}): \begin{array}{lllll}0 & 0.25 & 0.5 & 1 & 2\end{array}$ b

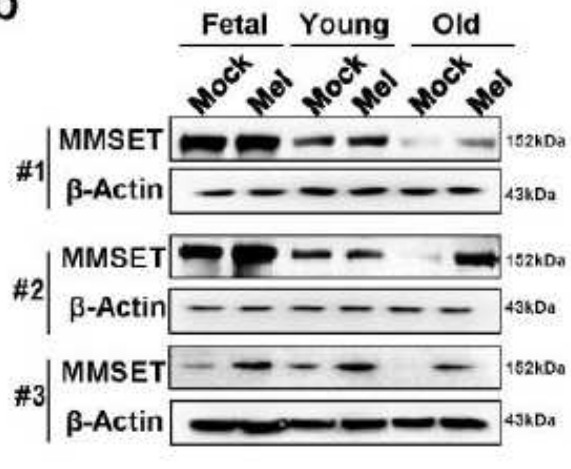

e
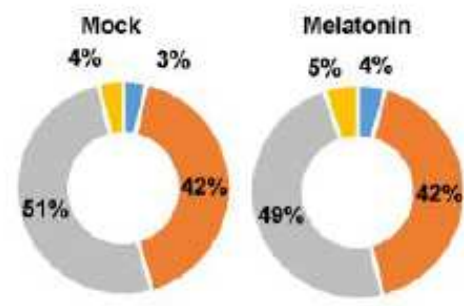

- promoter

* intragenic

- intergenic

others

C
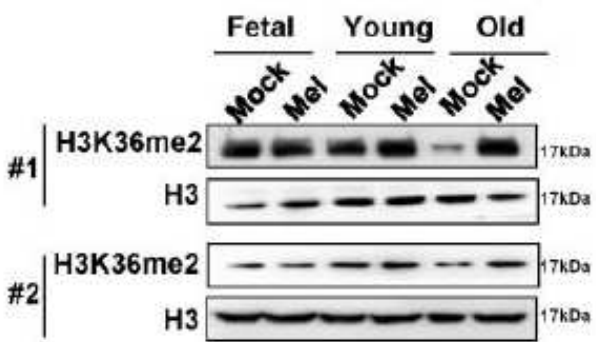

$\# 3\left|\begin{array}{r}\mathrm{H} 3 \mathrm{~K} 36 \mathrm{me} 2 \\ \mathrm{H} 3\end{array}\right|$

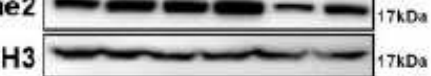

g

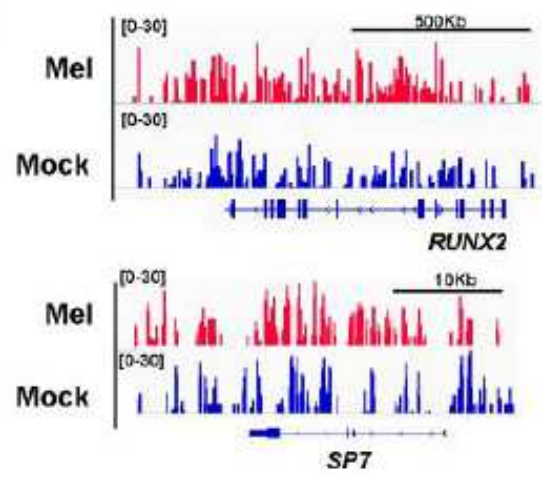

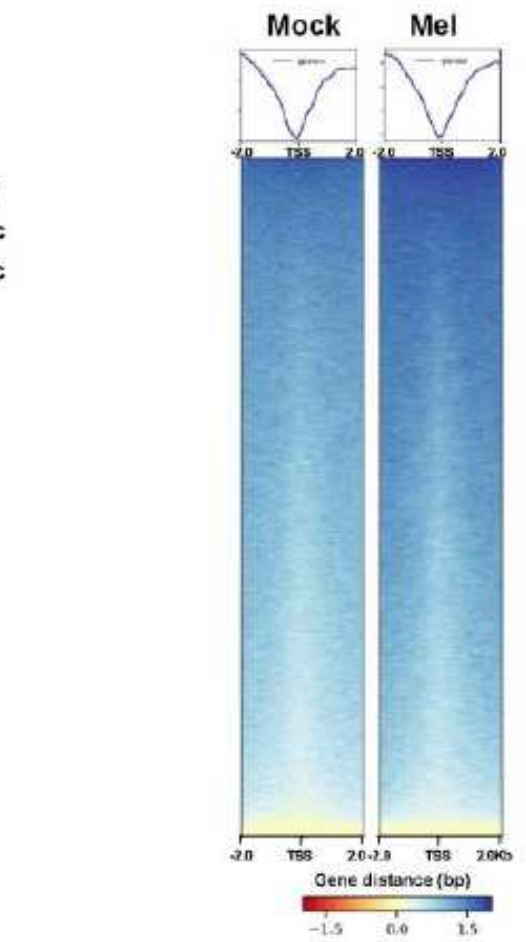

f

\section{Figure 4}

Melatonin stimulates MMSET expression in senescent MSCs. (a) mRNA level of MMSET of MSCs from donors of different ages treated with melatonin ( $\mathrm{n}=12$ with each sample triplicated). n.s., no significance. (b) The MMSET expression in three primary MSCs from donors of different ages treated with melatonin. (c) The H3K36me2 levels in three primary MSCs from donors of different ages treated with melatonin. (d) mRNA levels of RUNX2 and SP7/OSTERIX in MSCs treated with increasing dosage of melatonin for 24 hours $\left(n=3\right.$ with each detection triplicated). ${ }^{*}, P<0.05$; $* *, P<0.01$. (e) Pie chart for ChIP-seq profile to show percentage of genes enriched by H3K36me2 in MSC cells treated with vehicle or $1 \mu \mathrm{M}$ melatonin ( $\mathrm{n}=3$ ); ( $\mathrm{f}$ ) Changes of H3K36me2 density around the TSS region of genes. $(\mathrm{g})$ Representative ChIP-seq profiles for H3K36me2 occupancy at the promoter regions of RUNX2 and SP7/OSTERIX genes. All Western blot results represents a representative example of three independently performed studies, each yielding similar results. 
a
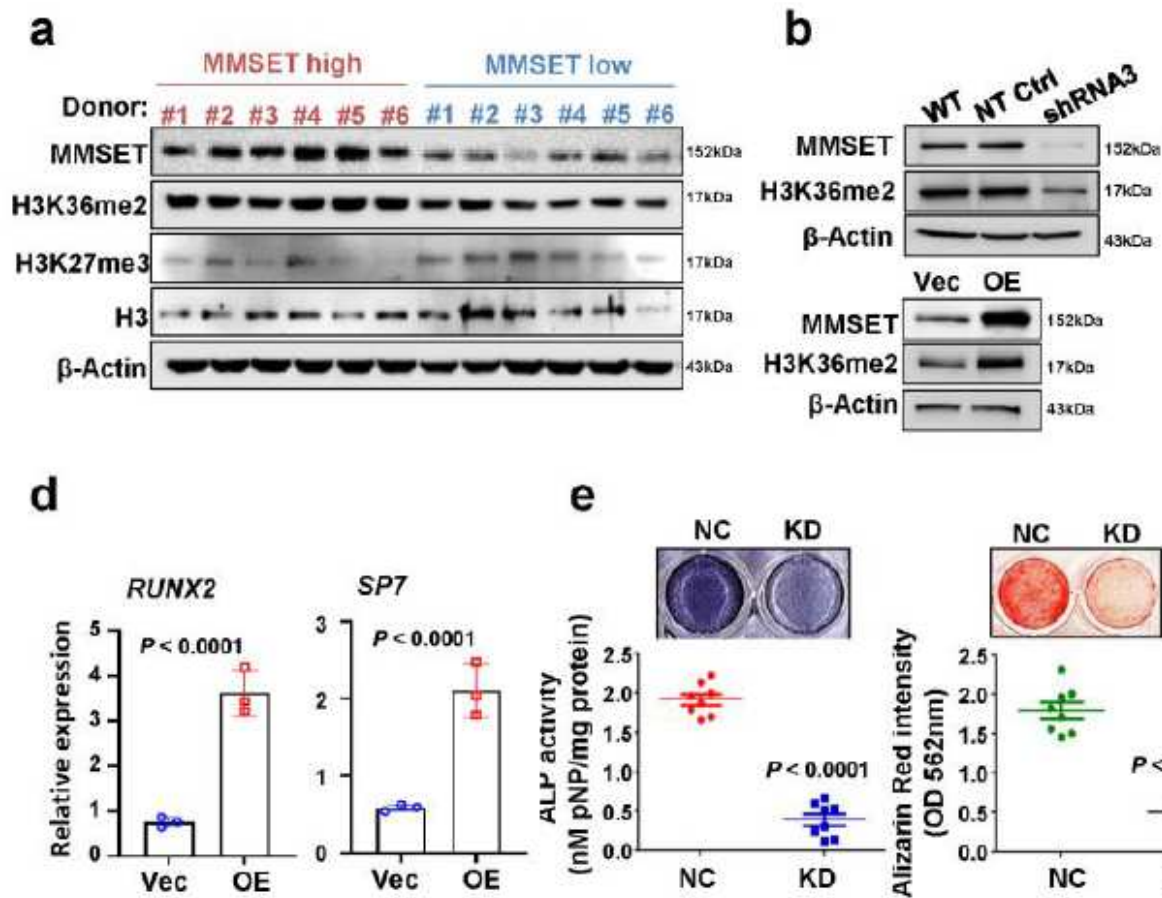

e

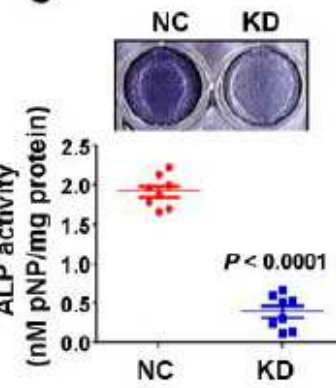

h
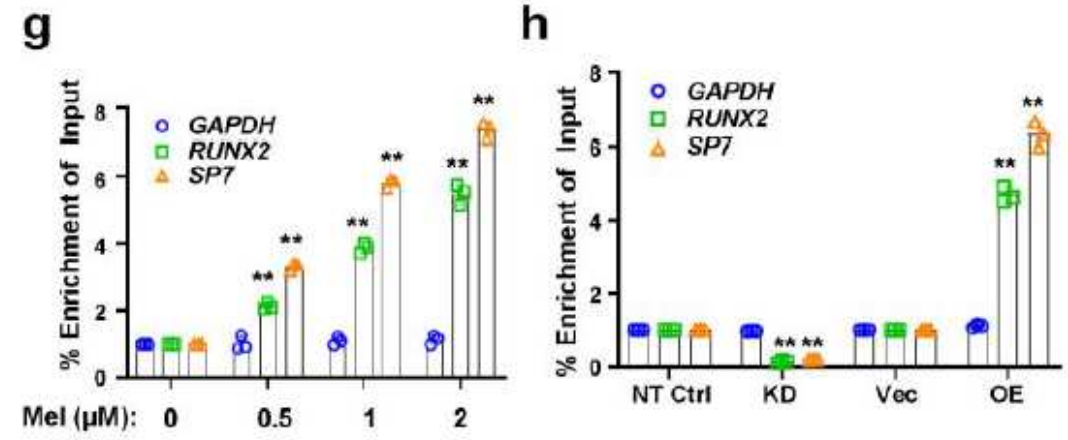
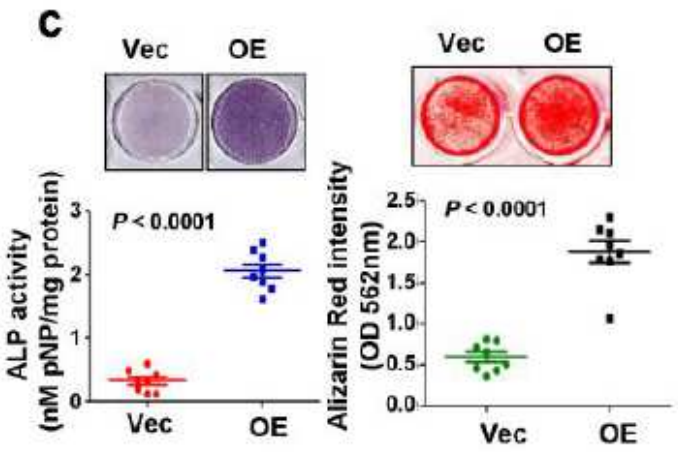

f
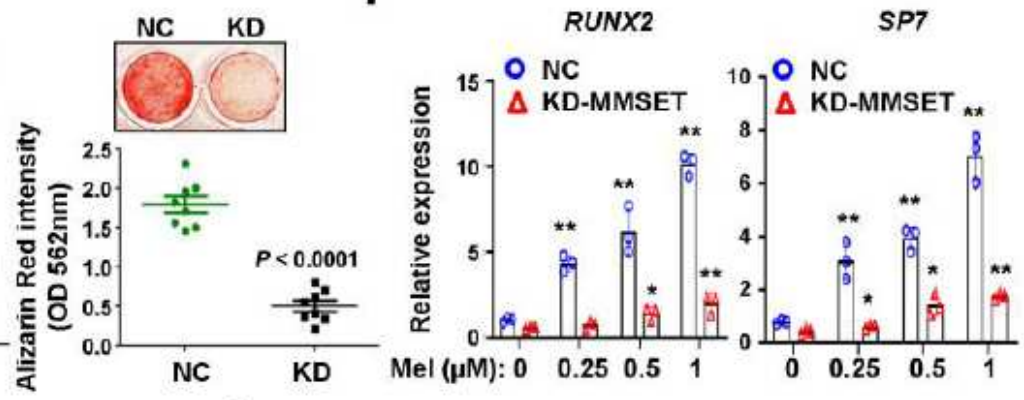

i

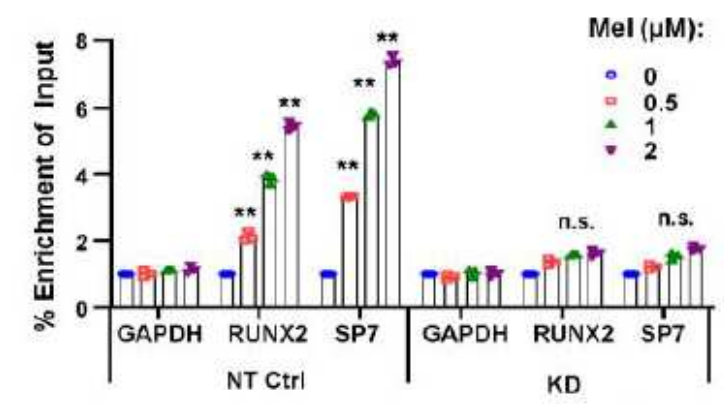

\section{Figure 5}

MMSET favors osteogenic differentiation. (a) Levels of MMSET, H3K36me2 and H3K27me3 in MSCs $(n=6)$. (b) Expression of MMSET and the corresponding H3K36me2 level in MSCs with MMSET knockdown (upper panel) or overexpression (lower panel). (c) The representative images (upper panel) and quantification (lower panel) of ALP and Alizarin Red S assay in MSCs with MMSET overexpression (n = 8). (d) Expressions of RUNX2 and SP7/OSTERIX in MSCs with MMSET overexpression ( $n=3$ with each detection triplicated). (e) The representative images (upper panel) and quantification (lower panel) of ALP and Alizarin Red S assay in MSCs with MMSET silencing $(n=8)$. (f) RUNX2 and SP7/OSTERIX expressions in MSCs with MMSET silencing treated with increasing amount of melatonin for 24 hours ( $\mathrm{n}$ $=3$ with each detection triplicated). ${ }^{*}, \mathrm{P}<0.05 ; * \star, \mathrm{P}<0.01 . \mathrm{g}, \mathrm{h}$ and $\mathrm{i}, \mathrm{ChIP-qPCR}$ analysis showed the recruitment of H3K36me2 on RUNX2 and SP7 promoter in MSCs treated with increasing dosage of melatonin for 24 hours (g), with overexpressed MMSET (h), or with MMSET silencing and then treated with increasing dosage of melatonin for 24 hours (i). The values represent mean \pm standard error of the mean (SEM) $\left(n=3\right.$ with each detection triplicated). n.s., no significance. ${ }^{\star}, P<0.01$. All Western blot results represents a representative example of three independently performed studies, each yielding similar results. 
a

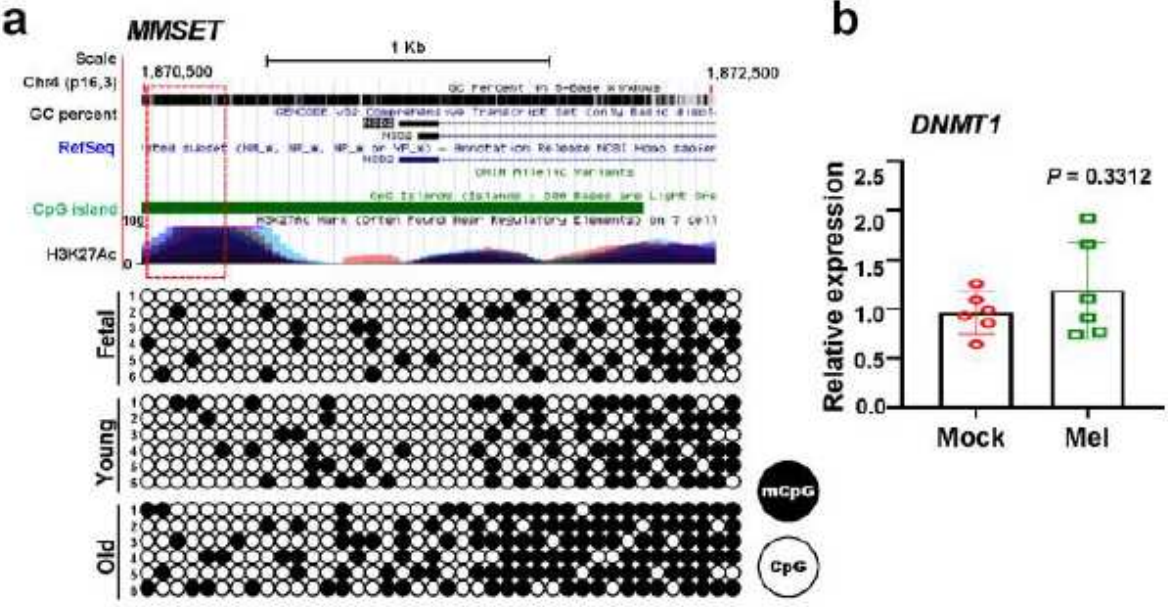

C

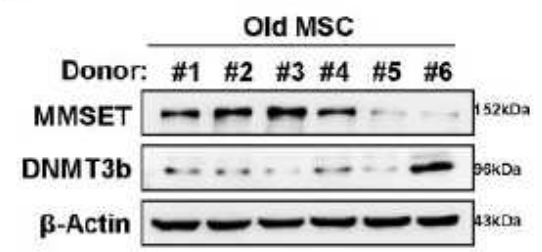

$\mathbf{f}$

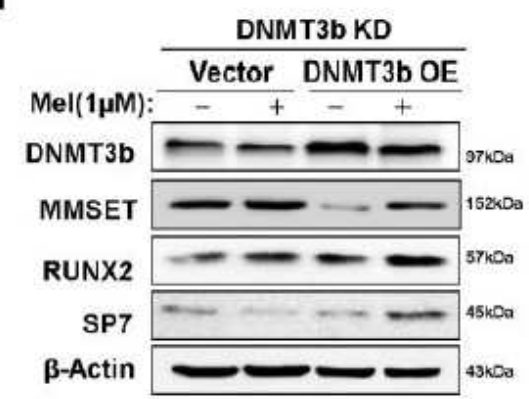

d

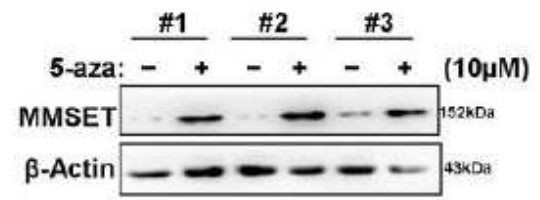

g

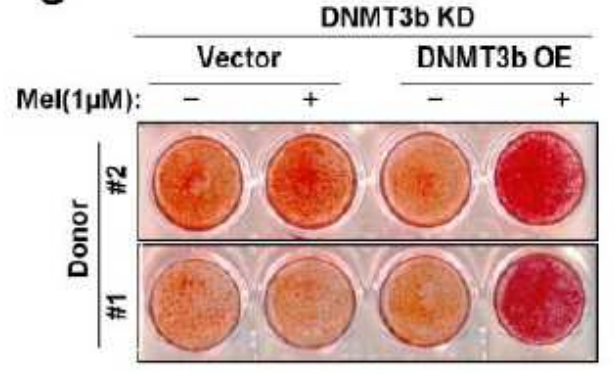

DNMT3a
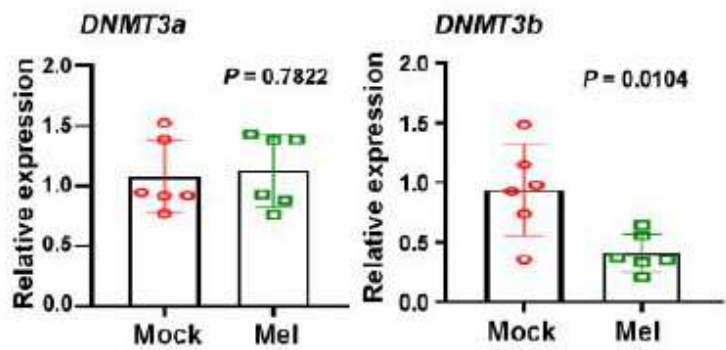

Figure 6

Melatonin alleviates DNA methylation of MMSET promoter. (a) Methylation signatures at an island on MMSET gene promoter in MSCs from different ages groups. (b) Expressions of DNMTs of senescent MSCs treated with $1 \mu \mathrm{M}$ melatonin for 24 hours determined by real time PCR $(n=6)$. (c) Expressions of MMSET and DNMT3b in senescent MSCs $(n=6)$. (d) MMSET expression in 3 senescent MSCs treated with $10 \mu \mathrm{M}$ 5-aza . (e) Expression of MMSET in MSCs with DNMT3b knockdown (KD) and treated with increasing dosage of melatonin for 24 hours. (f) DNMT3b, MMSET , RUNX2 and SP7 expression in the DNMT3b DNMT3b-knockdown MSCs with DNMT3b overexpression and treated with vehicle or $1 \mu \mathrm{M}$ melatonin. Representative images of Alizarin Red S assay (g) and ALP staining ( $h$ ) in the DNMT3bknockdown MSCs with DNMT3b overexpression and treated with vehicle or $1 \mu \mathrm{M}$ melatonin cultured with osteogenic media for 14 days. All Western blot results represents a representative example of three independently performed studies, each yielding similar results. 
a

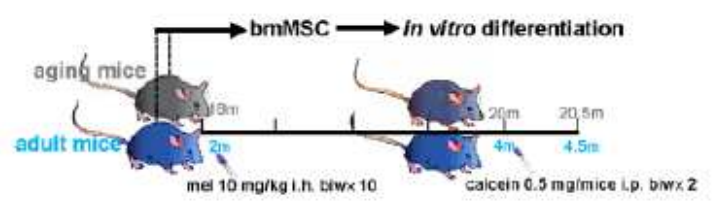

d

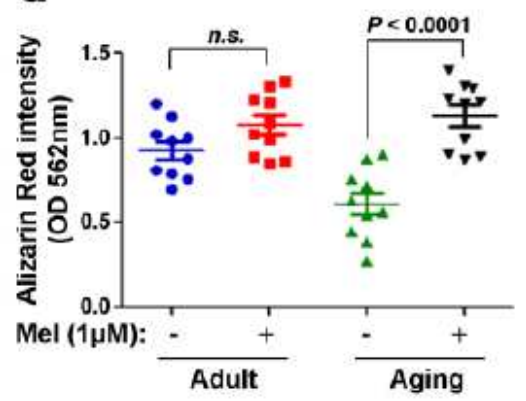

g

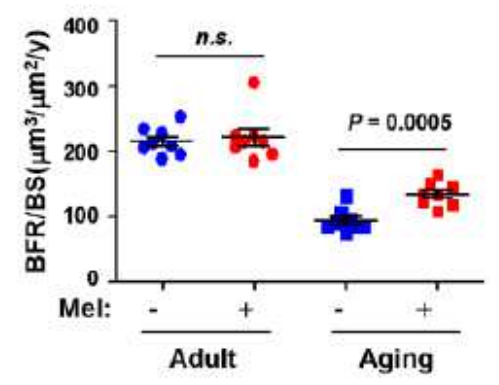

b

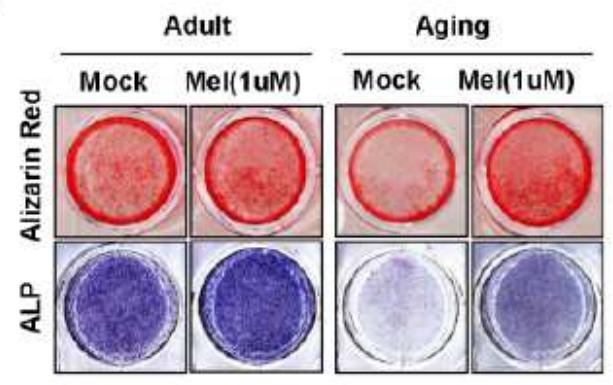

e

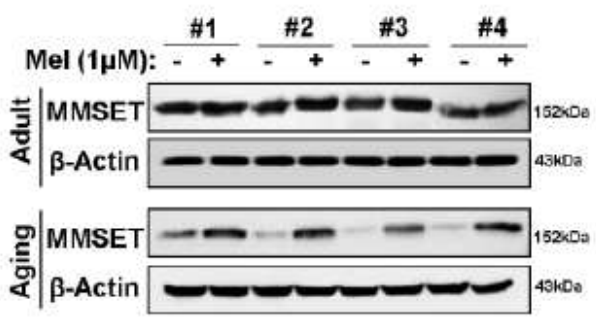

h

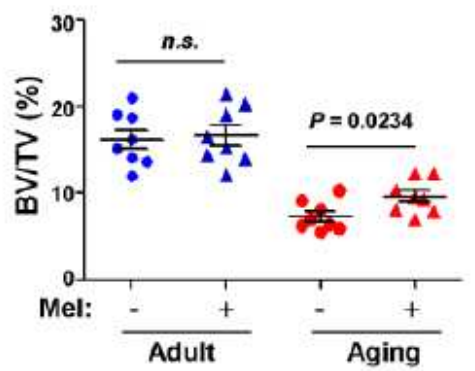

C

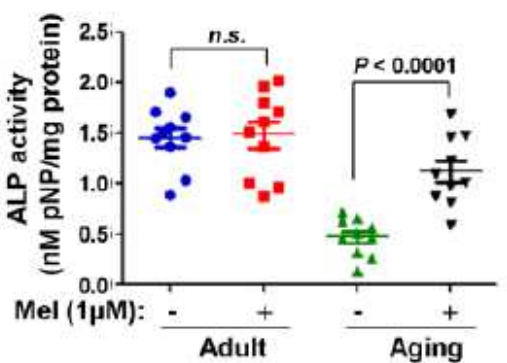

f

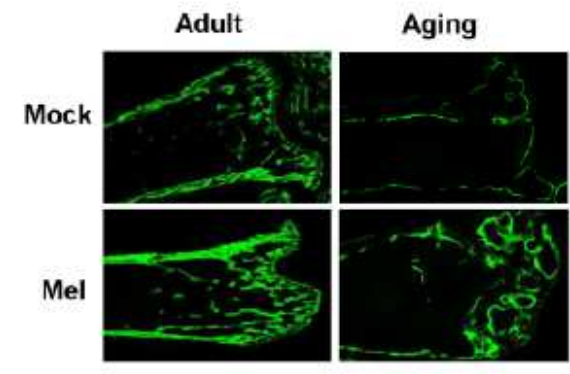

i

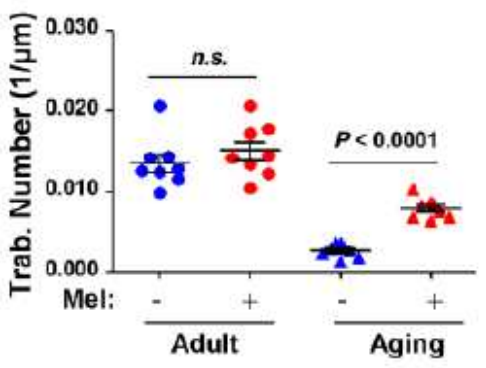

Figure 7

Melatonin facilitates osteogenesis of MSCs from aging mice via MMSET. (a) Treatment schedule and experiment design in aging mice and adult $m$ mice . (b) Representative images of ALP and Alizarin Red S staining of MSCs cultured with osteogenic media for 14 days from aging and young mice in presence with vehicle or melatonin. Quantification of ALP (c) and Alizarin Red S staining (d) of MSCs from aging and young mice groups treated with vehicle or melatonin $(n=10)$. (e) Representative MMSET expression in MSCs from aging and young mice treated with vehicle or melatonin for 24 hours hours. (f) The representative images of calcein stained femur bone from aging and young mice treated with vehicle or melatonin for 10 weeks. Quantification of bone formation rate (BFR/BS) (g), the percentage of bone volume to total volume (BV/TV) h) and number of bone trabecula (i) in femur femurs of aging and young mice treated with vehicle or melatonin $(n=8)$. n.s., no significance All Western blot results represents a representative example of three independently performed studies, each yielding similar results. 
a

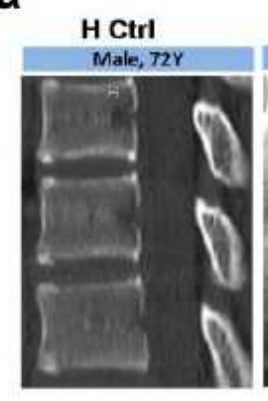

d

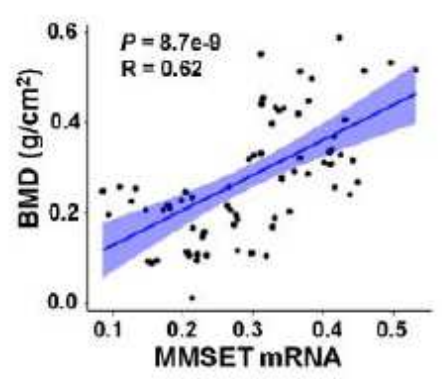

b

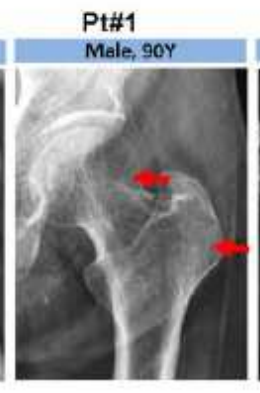

e
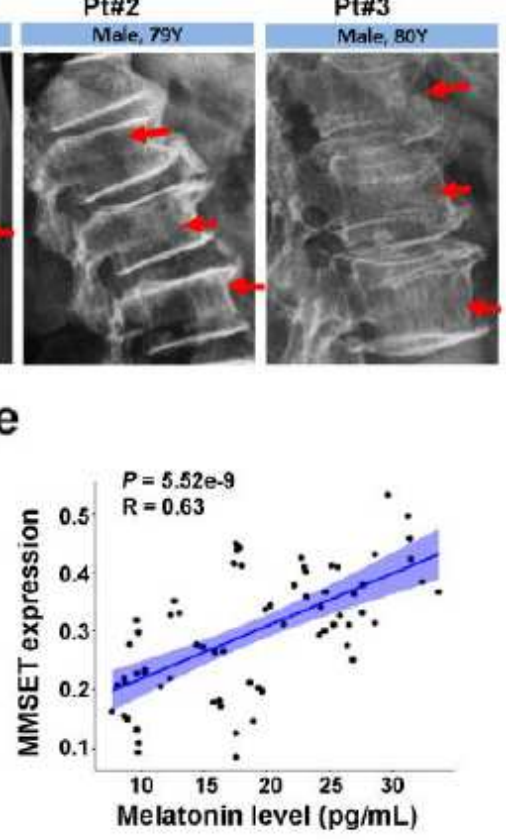

C

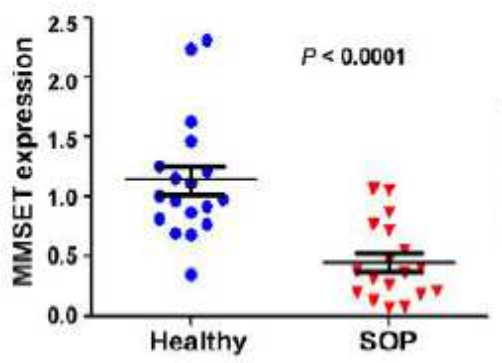

f

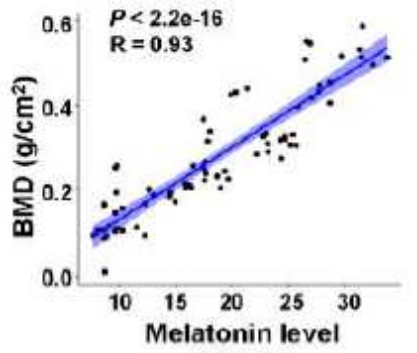

$(\mathrm{pg} / \mathrm{mL})$
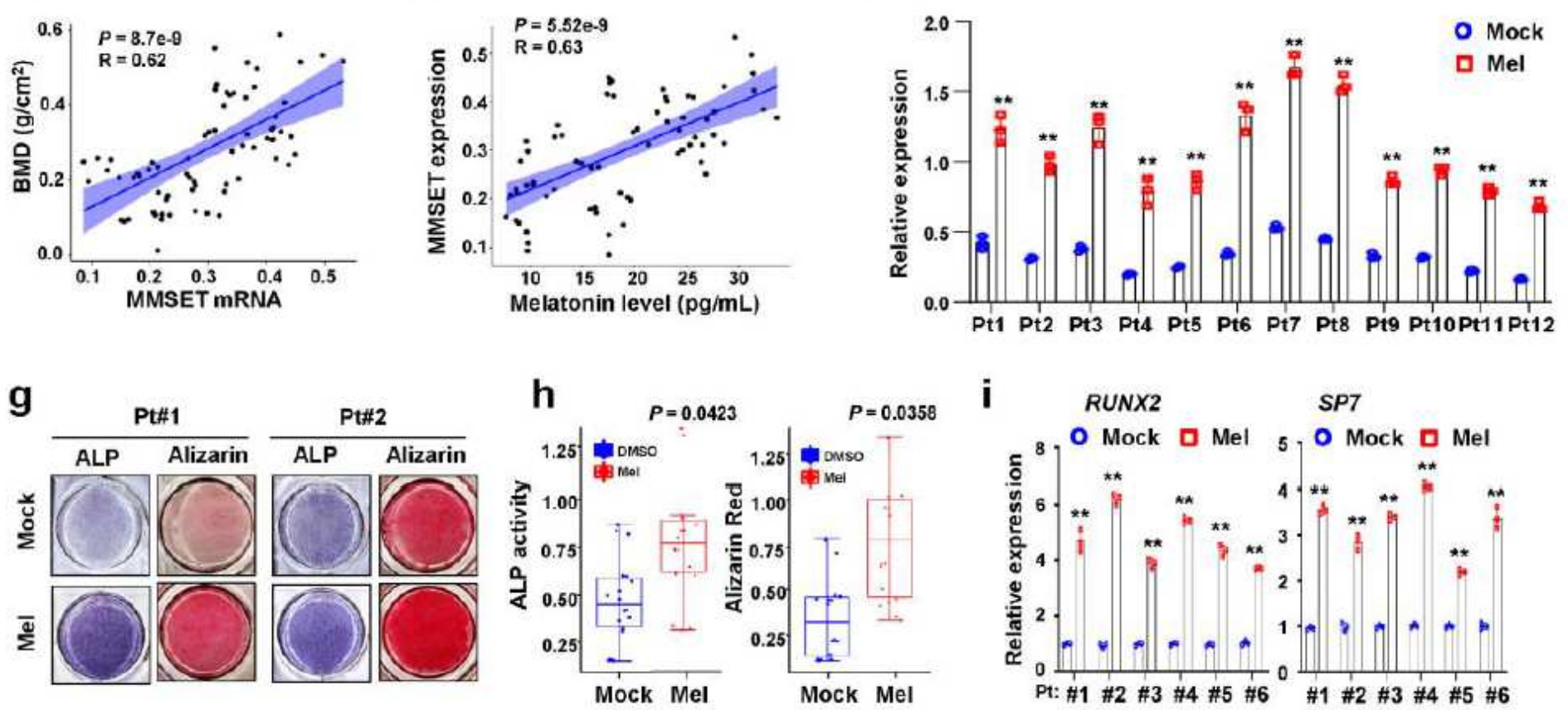

Figure 8

Melatonin treatment recovers osteogenic potential of MSCs from patients with senile osteoporosis osteoporosis. (a) Radiographic images of healthy control ( $\mathrm{H} \mathrm{Ctrl)}$ and patients with senile osteoporosis (Pt) Pt). Red arrows indicate the typical area with osteoporosis. (b) MMSET expression in MSCs isolated from healthy control and patients with senile osteoporosis ( $n=18$ with each detection triplicated). Correlation of bone mass density (BMD) with melatonin level in bone marrow plasma ( c), and MMSET expression in MSCs from senile osteoporosis patients (d) (3 independent reads for $\mathrm{n}=12$ patients)patients). (e)Correlation of MMSET expression in MSCs and melatonin level in bone marrow marrow plasma of patients with senile osteoporosis (3 independent reads for $n=12$ patients)patients). (f) MMSET expression in 12 MSCMSCs from patients with senile osteoporosis treated with $1 \mu \mathrm{M}$ melatonin for 24 hours ( $n=3$ with each detection triplicated ). **, $P<0.01$. (g) The representative images of ALP and Alizarin Red S staining in MSCs from donors with senile osteoporosis treated with osteogenic media for 14 days in presence of DMSO vehicle or melatonin melatonin. (h) Quantification of ALP and Alizarin Red S staining for MSCs under osteogenesis induction and in presence of DMSO vehicle or melatonin ( 3 independent reads for $n=6$ MSCs). (i) mRNA levels of RUNX2 and SP7SP7/OSTERIX in 
MSCs under osteogenesis induction and in presence of DMSO vehicle or melatonin ( $n=3$ with each detection triplicated ). ${ }^{\star *}, \mathrm{P}<0.01$.

\section{YOUNG}
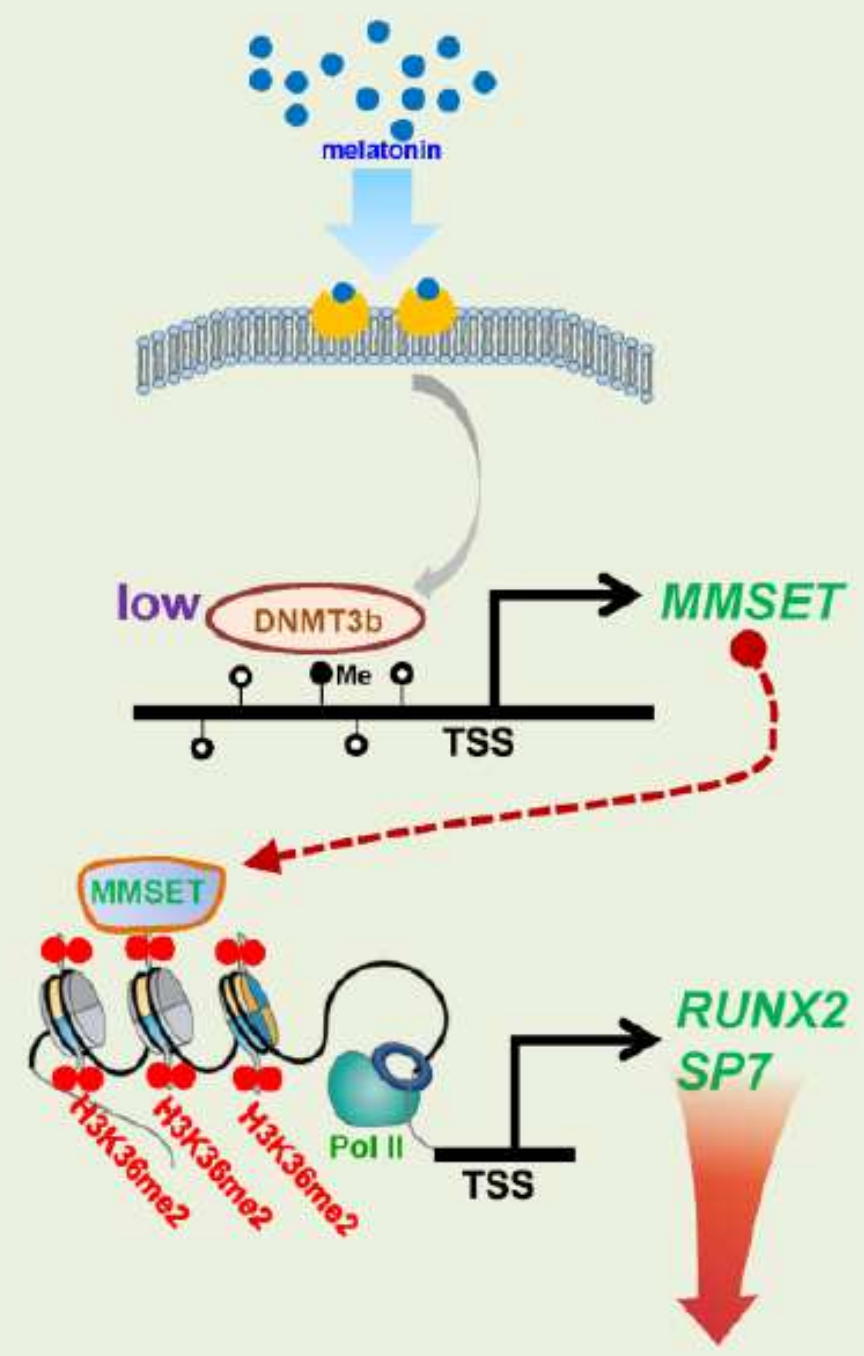

OB
OLD
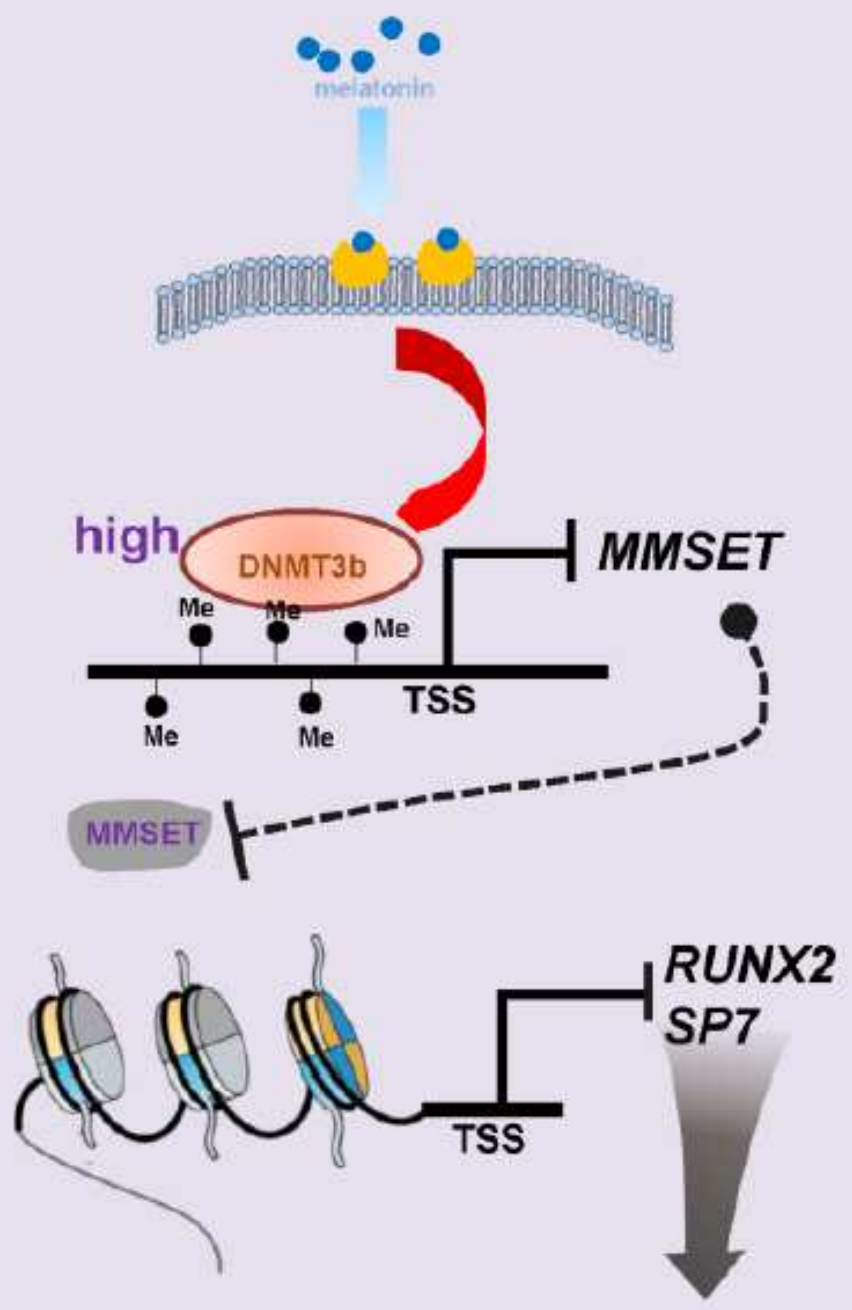

\section{Figure 9}

Proposed schematic diagram for mechanisms of promoting osteoblastogenesis from senescent BMSCs by melatonin: melatonin alleviates the senescence related hypermethylation of the MMSET promoter , upregulates expression of the histone methyltransferase NSD2; NSD2 promotes the histone H3 dimethylation modification at lysine 36 of the osteogenic genes RUNX2 and SP7/OSTERIX as a result, and consequentially enhances osteoblastogenesis from BMSCs

\section{Supplementary Files}

This is a list of supplementary files associated with this preprint. Click to download. 
- Supplementarymethods.docx

- SourceDataforallanalysis.xIsx

- supplementaryfigures.docx

- OriginalWesternblots.docx 